\title{
Loop quantum gravity and light propagation
}

\author{
Jorge Alfaro $^{a *}$, Hugo A. Morales-Técotl ${ }^{b c \dagger}$ \\ and Luis F. Urrutia ${ }^{d \S}$ \\ ${ }^{a}$ Facultad de Física \\ Pontificia Universidad Católica de Chile \\ Casilla 306, Santiago 22, Chile \\ ${ }^{b}$ Center for Gravitational Physics and Geometry, Department of Physics \\ The Pennsylvania State University, 104 Davey Lab \\ University Park PA 16802, USA \\ ${ }^{c}$ Departamento de Física \\ Universidad Autónoma Metropolitana Iztapalapa \\ A.P. 55-534, México D.F. 09340, México \\ ${ }^{d}$ Departamento de Física de Altas Energías \\ Instituto de Ciencias Nucleares \\ Universidad Nacional Autónoma de México \\ A.P. 70-543, México D.F. 04510, México
}

August 14, 2018

\begin{abstract}
Within loop quantum gravity we construct a coarse-grained approximation for the Einstein-Maxwell theory that yields effective Maxwell equations in flat spacetime comprising Planck scale corrections.

†hugo@xanum.uam.mx

$\ddagger$ Associate member of Abdus Salam International Centre for Theoretical Physics, Trieste, Italy.

§urrutia@nuclecu.unam.mx
\end{abstract}

*jalfaro@fis.puc.cl 
The corresponding Hamiltonian is defined as the expectation value of the electromagnetic term in the Einstein-Maxwell Hamiltonian constraint, regularized a la Thiemann, with respect to a would-be semiclassical state. The resulting energy dispersion relations entail Planck scale corrections to those in flat spacetime. Both the helicity dependent contribution of Gambini and Pullin [9] and, for a value of a parameter of our approximation, that of Ellis et. al. [10 are recovered. The electric/magnetic asymmetry in the regularization procedure yields nonlinearities only in the magnetic sector which are briefly discussed. Observations of cosmological Gamma Ray Bursts might eventually lead to the needed accuracy to study some of these quantum gravity effects. 


\section{Introduction}

It has been recently suggested that quantum gravity effects might be indeed observable [1]-[3]. Among the diverse phenomena where these observations could take place we can distinguish (see e.g. [4]-[0] ): (i) strain noise induced in gravitational wave detectors, (ii) neutral Kaon systems and (iii) energy dependent time of arrival of photons/neutrinos from distant sources. Experimental sensitivities in each of the above situations can be argued to be at the edge of what is required to reveal quantum gravity phenomena, namely Planck length sensitivities. In this work we focus on the third possibility. The idea is to look for modified dispersion relations of photons with energy $E$ and momentum $\vec{k}$, of the form

$$
c^{2} \vec{k}^{2}=E^{2}\left(1+\xi \frac{E}{E_{Q G}}+\mathcal{O}\left(\frac{E}{E_{Q G}}\right)^{2}\right),
$$

where $\xi$ is a numerical factor of order one and $E_{Q G}$ is an energy scale of order $\leq 10^{19} \mathrm{GeV}$, which signals the need of considering the quantum character of gravity. The above expression leads to the following modification of the speed of light in vacuum

$$
v=\frac{\partial E}{\partial k}=c\left(1-\xi \frac{E}{E_{Q G}}+\mathcal{O}\left(\frac{E}{E_{Q G}}\right)^{2}\right),
$$

which implies a retardation time

$$
\Delta t \approx \xi \frac{E}{E_{Q G}} \frac{L}{c},
$$

with respect to a signal propagating with speed $c$. When we consider cosmological distances $L \approx 10^{10}$ ly and an energy scale $E_{Q G} \approx 10^{19} \mathrm{GeV}$ in (3), the corresponding values are $\Delta t \approx$ $10^{-3} \mathrm{~s}(E \approx 20 \mathrm{MeV})$ and $\Delta t \approx 10^{-5} \mathrm{~s}(E \approx 0.20 \mathrm{MeV})$. In order to detect such effects, an experimental time resolution $\delta t$ at least of order $\Delta t$ is required. Thus, short and intense bursts traveling large distances would be the best candidates. Recent observations pointing towards the possibility of attaining such conditions are: (i) some Gamma Ray Bursts (GRB) originate 
at cosmological distances, $\left(\sim 10^{10} \mathrm{ly}\right)[6]$ and (ii) sensitivities $\delta t$ up to submillisecond scale have been achieved in GRB observations [7] and they are expected to improve in future spatial experiments [8].

It is thus timely to investigate whether candidate quantum gravity theories can account for (1). Modifications to Maxwell's equations in vacuum, induced by quantum gravity effects, have been calculated by Gambini and Pullin [9]. By considering a semiclassical regime in which the electromagnetic field is a classical object whereas space is described by loop quantum gravity, they obtained the dispersion relations

$$
\omega_{ \pm}(k)=k\left(1 \mp 2 \xi \ell_{P} k\right), \quad k=|\vec{k}|
$$

where \pm labels the helicity of the photon and $\ell_{P} \approx 10^{-33} \mathrm{~cm}$ is the Planck length. This modification, being helicity dependent, yields parity violation and birefringent effects.

On the other hand, a string theory approach proposed by Ellis et al. [10 suggests that a D-brane recoil in the quantum-gravitational foam induces a distortion in the surrounding space, which modifies the photon propagation properties. For a review of this approach see for example Ref. [11]. The dispersion relations obtained via this second procedure are

$$
\omega(k)=k\left(1-\xi \frac{k}{M_{D}}\right),
$$

with $\xi>0$. They arise from parity conserving corrections to Maxwell's equations which lead to a first order ( in $1 / M_{D}$ ) helicity independent effect in the dispersion relations that is linear in the photon energy. No birefringent effects appear at this order. In this approach, the red-shifted difference in the time arrival of two photons with present-day energies $E_{1}$ and $E_{2}$ has been calculated. For the BATSE data, when the redshifts $z$ of the GRB are known, a small subset of coincident photon pulses corresponding to channel 1 (20-50 keV) and channel $3(100-300 \mathrm{keV})$ are fitted and $\Delta t$ is calculated [10]. No significant effect in the data available is found. On the other hand, none of the pulses studied exhibited microbursts having a short time structure on scales $\leq 10^{-2}$ s. Were this the case, the sensitivity of the analysis would be 
greatly improved. Alternative studies based upon effective perturbative quantum gravity [12], open system techniques [13] and quantum light cone fluctuations [14] have been performed.

The study of cosmological neutrinos could also provide an excellent arena to probe quantum gravity induced propagation effects because space is practically transparent to them, even at very high energies. In fact, the fireball model, which is one of the most popular models of GRB, predicts the generation of $10^{14}-10^{19} \mathrm{eV}$ Neutrino Bursts (NB) [15, [16]. The planned Neutrino Burster Experiment (NuBE) will measure the flux of ultra high energy neutrinos (>10 TeV) over a $\sim 1 \mathrm{~km}^{2}$ effective area, in coincidence with satellite measured GRB's [17]. It is expected to detect $\approx 20$ events per year, according to the fireball model. Other relevant experiment aimed at observing ultra high energy cosmic rays, including neutrinos, is the OWL/Airwatch project which expects to see $\sim 3 \times 10^{3}-10^{5}$ cosmic ray events with energies $>10^{20} \mathrm{eV}[18$, 19]. Notably, this experiment is able to investigate time correlations among high energy neutrinos and gamma-rays. Hence in the foreseeable future, it might be possible to study quantum gravity effects on observed astrophysical neutrinos and photons. At the least, such observations could be used to restrict quantum gravity theories.

Motivated by these interesting possibilities, we have calculated the quantum gravity induced modifications to neutrino propagation in [20], within the loop quantum gravity framework. We obtained corrections to the velocity of propagation which are proportional to $\left(k \ell_{P}\right)$ together with helicity dependent corrections of order $\left(k \ell_{P}\right)^{2}$. The energy dependence in the first case coincides with that found later by Ellis et. al. using string theory methods [21].

In this work we extend our approach to the case of photons. The corrections obtained within our approximation contain those of [9] and, for a given value of a parameter in our scheme, those of [10]. Besides, we briefly discuss higher order nonlinearities arising in the magnetic sector of the effective Maxwell Hamiltonian.

The organization of the paper is as follows. In section 2 we recall some basic aspects of loop quantum gravity which are necessary for our analysis. After reviewing Thiemann's regularization of the Hamiltonian constraint of the Einstein-Maxwell theory in section [ : we 
provide a general description of our approximation in section 1 . The corrections arising from the electric and magnetic sectors are calculated in section 5. Once the effective Hamiltonian is obtained, we derive the modified Maxwell equations together with the corresponding dispersion relations in section 6. Section 7 contains a brief analysis of the non-linear effects arising from the magnetic sector. An outline of red shift effects on cosmological photon time delays is given in section 8. Finally, section 9 contains a discussion of the results.

\section{Loop quantum gravity}

In this section we summarize the main ingredients defining this approach also denominated quantum geometry [22]. Among the main results along this approach one finds: i) well defined geometric operators possessing a discrete spectrum, thus evidencing discreteness of space [22], ii) a microscopic account for black hole entropy [23] and, more recently, hints on quantum avoidance of a would be classical cosmological singularity [24]. (For a review on these topics see for example Ref. [25].)

To begin with it is assumed that the spacetime manifold $M$ has topology $\Sigma \times \mathbb{R}$, with $\Sigma$ a Riemannian 3-manifold. Here a co-triad $e_{a}^{i}$ is defined, with $a, b, c, \ldots$ being spatial tensor indices and $i, j, k, \ldots$ being $s u(2)$ indices. Thus the corresponding three-metric is given by $q_{a b}=e_{a}^{i} e_{b}^{i}$. In addition, a field $K_{a}^{i}$ is defined by $K_{a b}=\operatorname{sgn}\left[\operatorname{det}\left(e_{c}^{j}\right)\right] K_{a}^{i} e_{b}^{i}$, which is related to the extrinsic curvature $K_{a b}$ of $\Sigma$. A canonical pair for the gravitational phase space is $\left(K_{a}^{i}, E_{j}^{b} / \kappa\right)$,

where $E_{i}^{a}=\frac{1}{2} \epsilon^{a b c} \epsilon_{i j k} e_{b}^{j} e_{c}^{k}$ and $\kappa$ is Newton's constant. It turns out that such a canonical pair yields a complicated form for the Hamiltonian constraint of general relativity. A convenient canonical pair, making this constraint polynomial, was introduced by Ashtekar [26]. Nevertheless, two severe difficulties to proceed with the quantization remained: (i) the implementation of a diffeomorphism covariant regularization for the density-weight two Hamiltonian constraint hereby obtained and (ii) the extension to non-compact groups of the diffeomorphism covariant techniques already developed for gauge theories with compact groups [27]. In fact, the Ashtekar 
variables $\left({ }^{\mathbb{C}} A_{a}^{i}=\Gamma_{a}^{i}-i K_{a}^{i}, \quad i E_{i}^{a} / \kappa\right)$ [26], with $\Gamma_{a}^{i}$ being the torsion free connection compatible with $e_{a}^{i}$, are complex valued. Namely the gauge group is $S L\left(2, \mathscr{C}^{\prime}\right)$, which is non compact.

Some proposals to come to terms with difficulty (ii) were: to consider real connection variables [28], to implement a Wick transform [29] and to define tractable reality constraints [30]. All of these left open (i). Thiemann subsequently proposed to solve (i) and (ii) by incorporating real connection variables while keeping the density weight one character of the Hamiltonian constraint. He further provided a quantum version of the theory in the pure gravity case, as well as in those cases including the coupling of matter to gravity [31]. His approach is next reviewed, since we rely upon it for our analysis of the electromagnetic case.

Let us start with the following canonical pairs for the Einstein-Maxwell system: $\left(A_{a}^{i}=\right.$ $\left.\Gamma_{a}^{i}+K_{a}^{i}, E_{i}^{a} / \kappa\right)$ for the gravity sector and $\left(\underline{A}_{a}, \underline{E}^{a} / Q^{2}\right)$ for the electromagnetic sector. The latter has gauge group $U(1)$ and $Q$ is the electromagnetic coupling constant, related to the dimensionless fine structure constant by $\alpha_{E M}=Q^{2} \hbar$. The corresponding contributions to the Hamiltonian constraint are

$$
\begin{aligned}
& \left.H_{\text {Einstein }}[N]=\int_{\Sigma} d^{3} x N \frac{1}{\kappa \sqrt{\operatorname{det} q}} \operatorname{tr}\left(2\left[K_{a}, K_{b}\right]-F_{a b}\right)\left[E_{a}, E_{b}\right]\right), \\
& H_{\text {Maxwell }}[N]=\int_{\Sigma} d^{3} x N \frac{q_{a b}}{2 Q^{2} \sqrt{\operatorname{det} q}}\left[\underline{E}^{a} \underline{E}^{b}+\underline{B}^{a} \underline{B}^{b}\right] .
\end{aligned}
$$

Here $F_{a b}$ is the curvature of $A_{a}$ and $\underline{B}^{b}$ is the magnetic field of the $U(1)$ connection $\underline{A}$. The actual classical configuration space is the space $\mathcal{A} / \mathcal{G}$ of (both) connections modulo their gauge transformations. Indeed, this is what occurs in gauge theories where the fundamental field is a connection. This completes the classical description of the phase space of the theory.

The quantum arena is given as follows [27]. As in any quantum field theory, because of the infinite number of degrees of freedom, an enlargement of the classical configuration space is required. This is far from trivial since the measures defining the scalar product, which are required to provide a Hilbert space, get concentrated on distributional fields and hence lie outside the classical configuration space. The key idea to build up such an enlargement is to 
make Wilson loop variables (traces of parallel transport operators) well defined. The resulting space $\overline{\mathcal{A} / \mathcal{G}}$ can be thought of as the limit of configuration spaces of lattice gauge theories for all possible floating (i.e. not necessarily rectangular) lattices. Hence, geometric structures on lattice configuration space are used to implement a geometric structure on $\overline{\mathcal{A} / \mathcal{G}}$. This enables to define a background independent calculus on it which, in turn, leads to the construction of the relevant measures, the Hilbert space and the regulated operators.

In line with the Dirac procedure for constrained systems, one first ignores the constraints and constructs an auxiliary Hilbert space $\mathcal{H}_{\text {aux }}$, so that the set of elementary real functions on the full phase space is represented by self-adjoint operators in $\mathcal{H}_{\text {aux }}$. It turns out that $\mathcal{H}_{\text {aux }}$ is just $L^{2}\left(\overline{\mathcal{A} / \mathcal{G}}, \mu_{0}\right)$, with $\mu_{0}$ being a suitable measure that implements the self-adjointness property.

Diffeomorphism constraints are well defined operators on $\mathcal{H}_{\text {aux }}$ yielding no anomalies. There exists a dense subspace $\Phi$ of $\mathcal{H}_{\text {aux }}$ so that its topological dual $\Phi^{\prime}$ includes a complete set of solutions to the diffeomorphism constraint. They are characterized by generalized knots (i.e. diffeomorphism invariant classes of graphs). Besides, a diffeomorphism invariant Hilbert space is obtained for such states with an inner product that represents real observables by self-adjoint operators.

Furthermore, $\mathcal{H}_{\text {aux }}$ admits a basis in terms of the so called spin network states. A spin network is a triple $(\alpha, \vec{\jmath}, \vec{p})$ consisting of a graph $\alpha$, a coloring defined by a set of irreducible representations $\left(\jmath_{1}, \ldots, \jmath_{n}\right)$ of $S U(2)$, with $\jmath_{i}$ corresponding to the edge $e_{i}$ of $\alpha$ and a set of contractors $\left(p_{1}, \ldots, p_{m}\right)$. Here, a contractor $p_{k}$ is just an intertwining operator from the tensor product of representations of the incoming edges at the vertex $v_{k}$ to the tensor product of representations of the outgoing edges. Compactness of $S U(2)$ makes the vector space of all possible contractors $p_{k}$ finite, for a given $\vec{\jmath}$ and vertex $v_{k}$. An additional non-degeneracy condition is included: $j_{e}$ is not trivial for any edge $e$ and $\alpha$ is taken to be minimal (i.e. any other $\alpha^{\prime}$, occupying the same points in $\Sigma$ as $\alpha$, can always be built by subdividing the edges of $\alpha$, but not the other way around). 
A spin network state is a $C^{\infty}$ cylindrical function (a function that depends on the connection at the finite number of edges of a graph) on $\overline{\mathcal{A} / \mathcal{G}}$, constructed from a spin network

$$
T_{\alpha, \vec{\jmath}, \vec{c}}[A]:=\left[\otimes_{i=1}^{n} \jmath_{i}\left(h_{e_{i}}(A)\right) \bullet \otimes_{k=1}^{m} p_{k}\right]
$$

for all $A \in \overline{\mathcal{A}}$, which includes distributional besides smooth connections. $h_{e_{i}}(A)$ is an element of $S U(2)$ associated with the edge $e_{i}$ and "•" stands for contracting, at each vertex $v_{k}$ of $\alpha$, the upper indices of the matrices corresponding to all the incoming edges and the lower indices of the matrices assigned to the outgoing edges, with all the indices of $p_{k}$.

Given a pair $\alpha, \vec{\jmath}$ the vector space generated by $T_{\alpha, \vec{\jmath}, \vec{p}}$, for all possible contractors associated with $\alpha, \vec{\jmath}$ in the way stated previously, is denoted by $\mathcal{H}_{\text {aux }}^{\alpha, \vec{\jmath}}$. Then

$$
\mathcal{H}_{\mathrm{aux}}=\oplus_{\alpha, \vec{\jmath}} \mathcal{H}_{\mathrm{aux}}^{\alpha, \vec{\jmath}}
$$

where $\alpha, \vec{\jmath}$ run over all the pairs consisting of minimal graphs and irreducible non trivial representation labelings. The sum is orthogonal and the spaces $\mathcal{H}_{\text {aux }}^{\alpha, \vec{\gamma}}$ are finite dimensional. It suffices to define an orthonormal basis within each of them.

Note that the aforementioned construction of $\mathcal{H}_{\text {aux }}$ holds actually for any diffeomorphism covariant theory of connections with compact gauge group. The choice of $S U(2)$ corresponds to the case of gravity described in terms of real connection variables. So the generalization we are interested in to include both gravity and the electromagnetic field is $\mathcal{H}=\mathcal{H}_{\text {aux }}^{S U(2)} \otimes \mathcal{H}_{\text {aux }}^{U(1)}$. The spin network states for the compound system are denoted by $T_{\alpha,[\vec{j}, \vec{p}],[\vec{c}, \vec{q}]}[A, \underline{A}]$, with $\vec{c}, \vec{q}$ labeling the $U(1)$ coloring and contractors, respectively.

To extract physical information we will further need a state describing a flat continuous space $\Sigma$ at scales much larger than the Planck length, but not necessarily so at distances comparable to Planck length itself. States of this kind were introduced under the name of weave [32 for pure gravity. Flat weave states $|W\rangle$, having a characteristic length $\mathcal{L}$, were first constructed by considering collections of Planck scale circles randomly oriented. If one probes distances $d>>\mathcal{L}$ the continuous flat classical geometry is regained, while for distances $d<<\mathcal{L}$ 
the quantum loop structure of space is manifest. In other words, one expects a behavior of the type $\left\langle W\left|\hat{q}_{a b}\right| W\right\rangle=\delta_{a b}+O\left(\frac{\ell_{P}}{\mathcal{L}}\right)$. It was soon realized that such states could not yield a non trivial volume due to the lack of self intersections [33]. Couples of circles, intersecting at a point, were also considered as specific models of weaves to overcome this defect [34]. With the recent advances on the kinematical Hilbert space $\mathcal{H}_{\text {aux }}$ it became clear that all proposed weaves were afflicted by two undesirable features. First, they are defined to be peaked at a specific (flat or curved) metric, but not at a connection. This is in contrast with standard semiclassical states in terms of coherent states, for example. Second, the known weave states do not belong either to $\mathcal{H}_{\text {aux }}$ or to a dense subspace of it [35]. It may be possible to come to terms with such difficulties by defining coherent states for diffeomorphism covariant gauge theories [36] or by implementing a genuine statistical geometry [37], for instance. Both approaches have recently achieved substantial progress.

Nonetheless, in order to extract physics, there is the alternative possibility of using just the main features that semiclassical states should have. Namely, peakedness on both geometry and connection together with the property that they yield well defined expectation values of physical operators. An advantage of this alternative is that one may elucidate some physical consequences before the full fledged semiclassical analysis is settled down. Indeed, such alternative may be considered as complementary, in the sense of hinting at possible features of semiclassical states which could be further elaborated. After its completion, a rigorous semiclassical treatment should tell us whether the results arising from this alternative turn out to hold or not. The weakness of the treatment resides on its generality, since no detailed features of the would be semiclassical states are used -as opposed, say, to the original weave states- and hence a set of numerical coefficients cannot be calculated. Evaluating them will be the task of the rigorous semiclassical treatment.

On top of the purely gravitational semiclassical states, a generalization is required to include matter fields. For our analysis it will just suffice to exploit the same aspects of peakedness and well defined expectation values, extended to include the case of the electromagnetic field. The 
semiclassical states here considered will describe flat space and a smooth electromagnetic field living in it. Such a state is denoted by $\mid W, \underline{\vec{E}}, \underline{\vec{B}}>$ and has a characteristic length $\mathcal{L}$. Since no detailed information is used on how the semiclassical state is constructed in terms of, say, a graph, as opposed to weave states, the present approach yields results relying only on the following assumptions: (i) peakedness of the states, (ii) well defined expectation values and iii) existence of a coarse-grained expansion involving ratios of the relevant scales of the problem: the Planck length $\ell_{P}$, the characteristic length $\mathcal{L}$ and the electromagnetic wavelength $\lambda$. States fulfilling such requirements are referred to as would be semiclassical states in the sequel.

\section{The Regularization}

Thiemann has put forward a consistent regularization procedure to define the quantum Hamiltonian constraint of general relativity on $\mathcal{H}_{\text {aux }}$, both for pure gravity and matter couplings [31]. The basis of his proposal is the incorporation of the volume operator as a convenient regulator, since its action upon spin network states is finite. We use his regularization for the Einstein-Maxwell theory, which naturally allows the semiclassical treatment here pursued.

Consider the electric part of (6). The identity $\frac{1}{\kappa}\left\{A_{a}^{i}, V\right\}=2 \operatorname{sgn}\left(\operatorname{det} e_{b}^{j}\right) e_{a}^{i}$ allows to rewrite it as

$$
\begin{aligned}
H^{E}[N]= & \frac{1}{2 \kappa^{2} Q^{2}} \lim _{\epsilon \rightarrow 0} \frac{1}{\epsilon^{3}} \int_{\Sigma} d^{3} x N(x) \frac{\left\{A_{a}^{i}(x), V\right\}}{2(\operatorname{det} q)^{\frac{1}{4}}(x)} \underline{E}^{a}(x) \\
& \times \int_{\Sigma} d^{3} y \chi_{\epsilon}(x, y) \frac{\left\{A_{b}^{i}(y), V\right\}}{2(\operatorname{det} q)^{\frac{1}{4}}(y)} \underline{E}^{b}(y), \\
= & \left.\frac{1}{2 \kappa^{2} Q^{2}} \lim _{\epsilon \rightarrow 0} \int_{\Sigma} d^{3} x N(x)\left\{A_{a}^{i}(x), \sqrt{V(x, \epsilon)}\right)\right\} \underline{E}^{a}(x) \\
& \times \int_{\Sigma} d^{3} y \chi_{\epsilon}(x, y)\left\{A_{b}^{i}(y), \sqrt{V(y, \epsilon)}\right\} \underline{E}^{b}(y),
\end{aligned}
$$

with $\chi_{\epsilon}(x, y)=\Pi_{a=1}^{3} \theta\left(\epsilon / 2-\left|x^{a}-y^{a}\right|\right)$ being the characteristic function of a cube with volume $\epsilon^{3}$ centered at $x$ and $V(x, \epsilon):=\int d^{3} y \chi_{\epsilon}(x, y) \sqrt{\operatorname{det} q}(y)$ being the volume of the box as determined 
by $q_{a b}$. Remarkably all $\epsilon$ dependence resides here. This is possible due to $H_{\text {Maxwell }}$ having density weight one and it is achieved at the price of explicitly breaking diffeomorphism covariance. This is harmless as far as diffeomorphism covariance is regained once the regulator is removed. This is the case indeed [31]. Next, let $\Sigma$ be triangulated into tetrahedra $\Delta$. Hence, the integral over $\Sigma$ in (9) is just a sum over the contributions of each tetrahedron $\Delta$.

The form of (9) suggests to focus upon the term inside each integral. As we will see below, this indeed simplifies the analysis. Let

$$
\begin{aligned}
\Theta^{i}[f] & :=\int d^{3} x f(x) \underline{E}^{a}(x)\left\{A_{a}^{i}(x), \sqrt{V(x, \epsilon)}\right\} \\
& =\sum_{\Delta} \int_{\Delta} d^{3} x f(x) \underline{E}^{a}(x)\left\{A_{a}^{i}(x), \sqrt{V(x, \epsilon)}\right\} \\
& =\sum_{\Delta} \int_{\Delta} f(x) e(x) \wedge\left\{A^{i}(x), \sqrt{V(x, \epsilon)}\right\} \\
\Theta^{i}[f] & =: \sum_{\Delta} \Theta_{\Delta}^{i}[f] .
\end{aligned}
$$

Also let us use the dual of $\underline{E}: e_{b c}:={ }^{*} \underline{E}_{b c}=\frac{1}{1 !} \epsilon_{b c d} \underline{E}^{d}$. For a two-surface $S, \int_{S} e=\frac{1}{2} \int_{S} \underline{E}^{d} \epsilon_{b c d} d x^{b} \wedge$ $d x^{c}=\frac{1}{2} \int_{S} n_{d} \underline{E}^{d} \tilde{\epsilon}_{b c} d x^{b} \wedge d x^{c}=\int_{S} \underline{E}^{d} n_{d} \tilde{\epsilon}, \tilde{\epsilon}$ being the volume two-form. Hence $\Phi^{E}(S):=\int_{S} e$ is the flux of $\underline{E}^{a}$ through $S$. Recalling that

$$
\begin{aligned}
\operatorname{tr}\left(\tau_{i} h_{s_{L}}\left\{h_{s_{L}}^{-1}, \sqrt{V(x, \epsilon)}\right\}\right) & =\operatorname{tr}\left(\tau_{i} \tau_{m} \int_{0}^{1} d t \dot{s}_{L}^{-1 a}(t)\left\{A_{a}^{m}\left(s_{L}^{-1}(t)\right), \sqrt{V(x, \epsilon)}\right\}\right)+\ldots \\
& =-\frac{\delta_{i m}}{2} \int_{0}^{1} d t \dot{s}_{L}^{-1 a}(t)\left\{A_{a}^{m}\left(s_{L}^{-1}(t)\right), \sqrt{V(x, \epsilon)}\right\}+\ldots \\
& \approx-\frac{1}{2} s_{L}^{a}(1)\left\{A_{a}^{i}\left(s_{L}^{-1}(0)\right), \sqrt{V(x, \epsilon)}\right\}
\end{aligned}
$$

and that, for small tetrahedra, $\Phi^{E}\left(F_{J K}\right) \approx \frac{1}{2} \epsilon_{a b c} s_{J}^{b}(\Delta) s_{K}^{c}(\Delta) \underline{E}^{a}$, it follows

$$
\begin{aligned}
& f(v) \epsilon^{J K L} \Phi^{E}\left(F_{J K}\right) \operatorname{tr}\left(\tau^{i} h_{s_{L}(\Delta)}\left\{h_{s_{L}(\Delta)}^{-1}, \sqrt{V(v(\Delta), \epsilon)}\right\}\right) \\
\approx & -\frac{1}{4} f(v) \epsilon^{J K L} \epsilon_{a b c} s_{J}^{b}(\Delta) s_{K}^{c}(\Delta) \underline{E}^{a} s_{L}^{d}(\Delta)\left\{A_{d}^{i}\left(s_{l}^{-1}(0)\right), \sqrt{V(x, \epsilon)}\right\} \\
= & -\frac{3 !}{2} f(v) \operatorname{vol}(\Delta) \underline{E}^{a}\left\{A_{a}^{i}\left(s_{l}^{-1}(0)\right), \sqrt{V(x, \epsilon)}\right\}
\end{aligned}
$$




$$
=-\frac{3 !}{2} \int_{\Delta} f e \wedge\left\{A^{i}(x), \sqrt{V(x, \epsilon)}\right\}
$$

We have then

$$
\Theta_{\Delta}^{i}[f]=-\frac{2}{3 !} f(v) \epsilon^{J K L} \Phi^{E}\left(F_{J K}\right) \operatorname{tr}\left(\tau^{i} h_{s_{L}(\Delta)}\left\{h_{s_{L}(\Delta)}^{-1}, \sqrt{V(v(\Delta), \epsilon)}\right\}\right),
$$

where we are denoting by $s_{J}(\Delta), s_{K}(\Delta), s_{L}(\Delta)$ the edges of the tetrahedra $\Delta$ having $v$ as common vertex. As stated, $F_{J K}$ is a surface parallel to the face determined by $s_{J}(\Delta), s_{K}(\Delta)$ which is transverse to $s_{L}(\Delta)$.

Hence

$$
H^{E}[N]=\frac{1}{2 \kappa^{2} Q^{2}} \lim _{\epsilon \longrightarrow 0} \sum_{\Delta \Delta^{\prime}} \Theta_{\Delta}^{i}[N] \Theta_{\Delta^{\prime}}^{i}[\chi] .
$$

Next one replaces $\underline{E}^{a}$ and $V(x, \epsilon)$ by its quantum counterparts and adapts the triangulation to the graph $\gamma$ corresponding to the state acted upon, in such a way that at each vertex $v$ of $\gamma$ and triplet of edges $e, e^{\prime}, e^{\prime \prime}$ a tetrahedron is defined with basepoint at the vertex $v(\Delta)=v$ and segments $s_{I}(\Delta), I=1,2,3$, corresponding to $s(e), s\left(e^{\prime}\right), s\left(e^{\prime \prime}\right)$, respectively [31. Here it is assumed that $\epsilon^{I J K} \epsilon_{a b c} s_{I}^{a} s_{J}^{b} s_{K}^{c} \geq 0$. The arcs connecting the end points of $s_{I}(\Delta)$ and $s_{J}(\Delta)$ are denoted $a_{I J}(\Delta)$, so that a loop $\alpha_{I J}:=s_{I} \circ a_{I J} \circ s_{J}^{-1}$ can be formed. Besides, the face spanned by the segments $s_{I}(\Delta)$ and $s_{J}(\Delta)$ is called $F_{I J}$.

The action of the regulated operator hereby obtained gets concentrated in the vertices of the graph, as expected from the explicit appearance of the volume operator. In successive steps we replace

$$
\begin{gathered}
\hat{\Theta}_{\Delta}^{i}[N]=-\frac{2}{3 !} \frac{1}{i \hbar} N(v(\Delta)) \epsilon^{J K L} \hat{\Phi}^{E}\left(F_{J K}\right) \operatorname{tr}\left(\tau^{i} h_{s_{L}(\Delta)}\left[h_{s_{L}(\Delta)}^{-1}, \sqrt{\hat{V}(v(\Delta), \epsilon)}\right]\right) \\
\hat{\Theta}_{\Delta^{\prime}}^{\prime i}[\chi]=-\frac{2}{3 !} \frac{1}{i \hbar} \chi_{\epsilon}\left(v(\Delta), v\left(\Delta^{\prime}\right)\right) \epsilon^{M N P} \hat{\Phi}^{E}\left(F_{M N}^{\prime}\right) \operatorname{tr}\left(\tau^{i} h_{s_{P}\left(\Delta^{\prime}\right)}\left[h_{s_{P}\left(\Delta^{\prime}\right)}^{-1}, \sqrt{\hat{V}\left(v\left(\Delta^{\prime}\right), \epsilon\right)}\right]\right)
\end{gathered}
$$

to obtain

$$
H^{E}[N]=-\frac{1}{\hbar^{2} 2 \kappa^{2} Q^{2}} \sum_{v \in V(\gamma)} N(v)\left(\frac{2}{3 !} \frac{8}{E(v)}\right)^{2} \sum_{v(\Delta)=v\left(\Delta^{\prime}\right)=v} \times
$$




$$
\begin{aligned}
& \times \operatorname{tr}\left(\tau^{i} h_{s_{L}(\Delta)}\left[h_{s_{L}(\Delta)}^{-1}, \sqrt{\hat{V}(v(\Delta), \epsilon)}\right]\right) \epsilon^{J K L} \hat{\Phi}^{E}\left(F_{J K}\right) \times \\
& \times \operatorname{tr}\left(\tau^{i} h_{s_{P}\left(\Delta^{\prime}\right)}\left[h_{s_{P}\left(\Delta^{\prime}\right)}^{-1}, \sqrt{\hat{V}\left(v\left(\Delta^{\prime}\right), \epsilon\right)}\right]\right) \epsilon^{M N P} \hat{\Phi}^{E}\left(F_{M N}^{\prime}\right) .
\end{aligned}
$$

The valence $n(v)$ of the vertex $v$ yields the contribution $E(v)=n(v)(n(v)-1)(n(v)-2) / 3$ ! of the adapted triangulation at each vertex of $\gamma$. Also, as $\epsilon \rightarrow 0, v(\Delta)=v\left(\Delta^{\prime}\right)$ are the only contributions left over. The final expression for the electric piece of the Hamiltonian constraint given in [31] is obtained by the explicit action of this operator on cylindrical functions. We refrain from doing that here because the form of the operator (16) is better suited for our approximation given below.

As for the magnetic part of $H_{\text {Maxwell }}$ we proceed similarly. Since

$$
\begin{aligned}
\underline{h}_{s} & =\mathrm{e}^{-i \int_{0}^{1} d t \dot{s}^{a}(t) \underline{A}_{a}(s(t))}=\underline{I}-i \int_{0}^{1} d t \dot{s}^{a}(t) \underline{A}_{a}(s(t))+\ldots \\
\left(\underline{h}_{\alpha_{J K}}-1\right) & =-i \int_{0}^{1} d t \dot{\alpha}_{J K}^{a}(t) \underline{A}_{a}(s(t))+\ldots \\
& =-i \int_{F_{J K}} \underline{B}^{a} d S_{a}+\ldots \\
& =-i \Phi^{B}\left(F_{J K}\right)+\ldots \\
& \approx-i \frac{1}{2} \epsilon_{a b c} s_{J}^{b}(1) s_{K}^{c}(1) B^{a}(v(\Delta))
\end{aligned}
$$

and

$$
\begin{aligned}
& f(v) \epsilon^{J K L}\left(\underline{h}_{\alpha_{J K}}-1\right) \operatorname{tr}\left(\tau_{i} h_{s_{L}(\Delta)}\left\{h_{s_{L}(\Delta)}^{-1}, \sqrt{V(x, \epsilon)}\right\}\right) \\
\approx & i \frac{1}{4} \epsilon^{J K L} \epsilon_{a b c} s_{J}^{b} s_{K}^{c} s_{L}^{d} f(v) B^{a}(v)\left\{A_{d}^{i}(v), \sqrt{V(x, \epsilon)}\right\} \\
= & i \frac{1}{2} \operatorname{vol}\left(s_{J}, s_{K}, s_{L}\right) \delta_{a}^{d} f(v) B^{a}(v)\left\{A_{d}^{i}(v), \sqrt{V(x, \epsilon)}\right\} \\
= & i \frac{3 !}{2} \operatorname{vol}(\Delta) f(v) B^{a}(v)\left\{A_{a}^{i}(v), \sqrt{V(x, \epsilon)}\right\} \\
= & i \frac{3 !}{2} \int_{\Delta} f(x) B^{I}(x) \wedge\left\{A^{i}(x), \sqrt{V(x, \epsilon)}\right\},
\end{aligned}
$$


we can write

$$
H^{B}[N]=\frac{1}{2 \kappa^{2} Q^{2}} \lim _{\epsilon \longrightarrow 0} \sum_{\Delta \Delta^{\prime}} \Xi_{\Delta}^{i}[N] \Xi_{\Delta^{\prime}}^{i}[\chi]
$$

where

$$
\Xi_{\Delta}^{i}[f]:=i \frac{2}{3 !} f(v) \epsilon^{J K L}\left(\underline{h}_{\alpha_{J K}}-1\right) \operatorname{tr}\left(\tau_{i} h_{s_{L}(\Delta)}\left\{h_{s_{L}(\Delta)}^{-1}, \sqrt{V(x, \epsilon)}\right\}\right) .
$$

Contrasting $\Xi_{I \Delta}^{i}$ with $\Theta_{I \Delta}^{i}$ we notice (i) different numerical factors: $i \frac{2}{3 !}$ for the former while $-\frac{2}{3 !}$ for the latter and (ii) to leading order, the magnetic flux $\epsilon^{J K L}\left(\underline{h}_{\alpha_{J K}}-1\right)$ has as its counterpart the electric flux $\epsilon^{J K L} E_{\alpha_{J K}}$.

The quantum version of the above operators is obtained using

$$
\hat{\Xi}_{\Delta}^{i}[f]:=i \frac{2}{3 !} \frac{1}{i \hbar} f(v) \epsilon^{J K L}\left(\underline{h}_{\alpha_{J K}(\Delta)}-1\right) \operatorname{tr}\left(\tau_{i} h_{s_{L}(\Delta)}\left[h_{s_{L}(\Delta)}^{-1}, \sqrt{V(x, \epsilon)}\right]\right) .
$$

Finally, one gets the regularized magnetic piece of the Hamiltonian constraint as [31]

$$
\begin{aligned}
H^{B}[N] & =+\frac{1}{\hbar^{2} 2 \kappa^{2} Q^{2}} \sum_{v \in V(\gamma)} N(v)\left(\frac{2}{3 !} \frac{8}{E(v)}\right)^{2} \sum_{v(\Delta)=v\left(\Delta^{\prime}\right)=v} \times \\
& \times \epsilon^{J K L} \operatorname{tr}\left(\tau_{i} h_{s_{L}(\Delta)}\left[h_{s_{L}(\Delta)}^{-1}, \sqrt{\hat{V}_{v}}\right]\right)\left(\underline{h}_{\alpha_{J K}(\Delta)}-1\right) \times \\
& \times \epsilon^{M N P} \operatorname{tr}\left(\tau_{i} h_{s_{P}\left(\Delta^{\prime}\right)}\left[h_{s_{P}\left(\Delta^{\prime}\right)}^{-1}, \sqrt{\hat{V}_{v}}\right]\right)\left(\underline{h}_{\alpha_{M N}\left(\Delta^{\prime}\right)}-1\right) .
\end{aligned}
$$

The electric and magnetic pieces of $H_{\text {Maxwell }}$ can be treated in a unified manner in terms of fluxes. To see this recall that for abelian gauge fields

$$
\underline{h}_{\alpha_{J K}(\Delta)}=e^{-i \int_{\alpha_{J K}(\Delta)} d t \dot{s}^{a}(t) \underline{\hat{A}}_{a}(s(t))}=e^{-i \hat{\Phi}^{B}\left(F_{J K}\right)},
$$

where $\hat{\Phi}^{B}\left(F_{J K}\right)$ is the flux of the magnetic field through the surface $F_{J K}$.

Then the full electromagnetic Hamiltonian is

$$
H_{\text {Maxwell }}[N]=+\frac{1}{\hbar^{2} 2 \kappa^{2} Q^{2}} \sum_{v \in V(\gamma)} N(v)\left(\frac{2}{3 !} \frac{8}{E(v)}\right)^{2} \sum_{v(\Delta)=v\left(\Delta^{\prime}\right)=v} \times
$$




$$
\begin{aligned}
& \times \operatorname{tr}\left(\tau_{i} h_{s_{L}(\Delta)}\left[h_{s_{L}(\Delta)}^{-1}, \sqrt{\hat{V}_{v}}\right]\right) \operatorname{tr}\left(\tau_{i} h_{s_{P}\left(\Delta^{\prime}\right)}\left[h_{s_{P}\left(\Delta^{\prime}\right)}^{-1}, \sqrt{\hat{V}_{v}}\right]\right) \\
& \times \epsilon^{J K L} \epsilon^{M N P}\left[\left(e^{-i \hat{\Phi}^{B}\left(F_{J K}\right)}-1\right)\left(e^{-i \hat{\Phi}^{B}\left(F_{M N}^{\prime}\right)}-1\right)-\hat{\Phi}^{E}\left(F_{J K}\right) \hat{\Phi}^{E}\left(F_{M N}^{\prime}\right)\right] .
\end{aligned}
$$

Let us emphasize the structure of the above regularized Hamiltonian. There is a common gravitational factor included in the $S U(2)$ trace. The basic entities that regularize the electromagnetic part are the corresponding fluxes: one is associated with the magnetic field, which enters through a product of exponential flux factors, while the other is related to the electric field, entering in a bilinear product of fluxes. Thus, in the quadratic field approximation the effective Hamiltonian preserves duality invariance. Nevertheless, the magnetic sector includes higher powers in the field expansion. Hence, nonlinearities in the field equations, inducing duality violations, arise only via the magnetic field.

Before proceeding let us recall that, according to Thiemann's conventions, in flat space we must have

$$
H_{\text {Maxwell }}=\int d^{3} x \frac{1}{2 Q^{2}}\left(\underline{E}^{a} \underline{E}^{a}+\underline{B}^{a} \underline{B}^{a}\right)
$$

where $Q$ is the electromagnetic coupling constant. The electromagnetic potential is denoted by $\underline{A}_{a}$ and the corresponding electromagnetic tensor by $\underline{F}_{a b}$. The units are such that the gravitational connection $A_{a}^{i}$ has dimensions of $1 / L$ (inverse Length) and the Newton's constant $\kappa$ has dimensions of $L / M$ (Length over Mass). Also we have that $\left[|\underline{\underline{E}}| / Q^{2}\right]=M / L^{3}$. Taking the dimensions of $\underline{A}_{a}$ to be $1 / L$, according to the corresponding normalization of the Wilson loop, we conclude that $[\underline{\vec{E}}]=[\underline{\vec{B}}]=1 / L^{2}$ and $\left[Q^{2}\right]=1 /(M L)$. In our case we also have $[\hbar]=M L$, which in fact leads to $\alpha_{E M}=Q^{2} \hbar$ to be the dimensionless fine-structure constant, as defined by Thiemann 31. 


\section{General structure of the calculation}

The effective Maxwell Hamiltonian is defined by considering the expectation value of the $U(1)$ gauge sector of the quantum Hamiltonian constraint with respect to $|W, \underline{\vec{E}}, \underline{\vec{B}}\rangle$. Inside this expectation value operators are expanded around all relevant vertices of the triangulation in powers of the segments $s_{L}^{a}(\Delta)$, which have lengths of order $\ell_{P}$. In this way, a systematic approximation is given involving the scales $\ell_{P} \ll \mathcal{L}<\lambda$, where $\lambda$ is the De Broglie wavelength of the photon. Our corrections to the Maxwell Hamiltonian arise from such an approximation.

We do the full calculation of the magnetic sector, including the non-linear contributions to order $\ell_{P}^{2}$. Next, to obtain the electric sector, it is enough to consider only the quadratic terms in the magnetic Hamiltonian and make the replacement $B \rightarrow E$.

In the case of the magnetic sector, the general form of the expectation value is (recalling that $\left.1 / \kappa^{2}=\hbar^{2} / \ell_{P}^{4}\right)$

$$
\begin{array}{r}
\mathrm{H}^{B}=-\frac{1}{2 Q^{2}} \frac{1}{\ell_{P}^{4}} \sum_{v \in V(\gamma)} N(v)\left(\frac{2}{3 !} \frac{8}{E(v)}\right)^{2} \sum_{v(\Delta)=v\left(\Delta^{\prime}\right)=v}<W, \underline{\underline{E}}, \underline{\vec{B}} \mid \underline{\hat{E}}_{p_{1} q_{1}}(v) \ldots \underline{\hat{E}}_{p_{n} q_{n}}(v) \\
\left(\partial^{a_{1}} \ldots \partial^{a_{m}} \underline{\hat{E}}_{p q}(v)\right) \hat{T}_{a_{1} \ldots a_{m}} p q p_{1} q_{1} \ldots p_{n} q_{n} \\
\left(v, s(\Delta), s\left(\Delta^{\prime}\right)\right) \mid W, \underline{\vec{E}}, \underline{\vec{B}}>.
\end{array}
$$

To proceed with the approximation we think of space as made up of boxes, each centered at a given point $\vec{x}$ and with volume $\mathcal{L}^{3} \approx d^{3} x$. Each box contains a large number of vertices of the semiclassical state $\left(\mathcal{L}>\ell_{P}\right)$, but is considered as infinitesimal in the scale where the

space can be regarded as continuous. Also, we assume that the magnetic operators are slowly varying inside the box $\left(\ell_{P}<<\lambda_{D}\right)$, in such a way that for all the vertices inside the box one can write

$$
\left\langle W, \underline{\vec{E}}, \underline{\vec{B}}\left|\ldots \underline{\hat{F}}_{a b}(v) \ldots\right| W, \underline{\vec{E}}, \underline{\vec{B}}\right\rangle=\mu \epsilon_{a b c} \underline{B}^{c}(\vec{x}) .
$$

Here $\underline{F}_{a b}=\partial_{a} \underline{A}_{b}-\partial_{b} \underline{A}_{a}, \underline{B}^{a}(\vec{x})$ is the classical magnetic field at the center of the box and $\mu$ is a dimensionless constant to be determined in such a way that we recover the standard classical 
result (23) in the zeroth order approximation. In the next section we show that

$$
\mu=\left(\frac{\mathcal{L}}{\ell_{P}}\right)^{1+\Upsilon},
$$

with $\Upsilon$ being a parameter defining the leading order contribution of the gravitational connection to the expectation value. Applying to (24) the procedure just described leads to

$$
\begin{aligned}
\mathrm{H}^{B}= & \sum_{\operatorname{Box}} N(\vec{x}) \underline{F}_{p_{1} q_{1}}(\vec{x}) \ldots \underline{F}_{p_{n} q_{n}}(\vec{x}) \ldots\left(\partial^{a_{1}} \ldots \partial^{a_{m}} \underline{F}_{p q}(\vec{x})\right) \sum_{v \in \mathrm{Box}} \ell_{P}^{3}\left(\frac{2}{3 !} \frac{8}{E(v)}\right)^{2} \\
& \sum_{v(\Delta)=v\left(\Delta^{\prime}\right)=v}\left(-\frac{1}{2 Q^{2}} \frac{\mu^{n+1}}{\ell_{P}^{4}}\right)<W, \underline{\vec{E}}, \underline{\vec{B}}\left|\frac{1}{\ell_{P}^{3}} \hat{T}_{a_{1} \ldots a_{m}}{ }_{p q p_{1} q_{1} \ldots p_{n} q_{n}}\left(v, s(\Delta), s\left(\Delta^{\prime}\right)\right)\right| W, \underline{\vec{E}}, \underline{\vec{B}}> \\
= & \sum_{\operatorname{Box}} N(\vec{x}) \underline{F}_{p_{1} q_{1}}(\vec{x}) \ldots \underline{F}_{p_{n} q_{n}}(\vec{x})\left(\partial^{a_{1}} \ldots \partial^{a_{m}} \underline{F}_{p q}(\vec{x})\right) d^{3} x T_{a_{1} \ldots a_{m}}{ }_{p q p_{1} q_{1} \ldots p_{n} q_{n}}(\vec{x}) \\
\mathrm{H}^{B}= & \int d^{3} x N(\vec{x}) \underline{F}_{p_{1} q_{1}}(\vec{x}) \ldots \underline{F}_{p_{n} q_{n}}(\vec{x})\left(\partial^{a_{1}} \ldots \partial^{a_{m}} \underline{F}_{p q}(\vec{x})\right) T_{a_{1} \ldots a_{m}}{ }_{p p_{1} q_{1} \ldots p_{n} q_{n}}(\vec{x}) .
\end{aligned}
$$

The box-averaged tensor $T_{a_{1} \ldots a_{m}} p q p_{1} q_{1} \ldots p_{n}(\vec{x})$, defined by

$$
\begin{aligned}
& T_{a_{1} \ldots a_{m}}{ }^{p} q p_{1} q_{1} \ldots p_{n} q_{n}(\vec{x})=\sum_{v \in \text { Box }}\left(\frac{2}{3 !} \frac{8}{E(v)}\right)^{2} \sum_{v(\Delta)=v\left(\Delta^{\prime}\right)=v}\left(-\frac{1}{2 Q^{2}} \frac{\mu^{n+1}}{\ell_{P}^{4}}\right) \\
& \times<W, \underline{\vec{E}}, \underline{\vec{B}}\left|\frac{1}{\ell_{P}^{3}} \hat{T}_{a_{1} \ldots a_{m}}{ }^{p q p_{1} q_{1} \ldots p_{n} q_{n}}\left(v, s(\Delta), s\left(\Delta^{\prime}\right)\right)\right| W, \underline{\vec{E}}, \underline{\vec{B}}>,
\end{aligned}
$$

is constructed from flat space tensors like $\delta_{a b}, \epsilon_{a b c}$. In this way we are demanding covariance under rotations at the scale $\mathcal{L}$.

When averaging inside each box, the scaling of the expectation values of the gravitational operators is estimated according to

$$
\begin{gathered}
\left\langle W, \underline{\vec{E}}, \underline{\vec{B}}\left|\ldots A_{i a} \ldots\right| W, \underline{\vec{E}}, \underline{\vec{B}}\right\rangle \approx \ldots \frac{1}{\mathcal{L}}\left(\frac{\ell_{P}}{\mathcal{L}}\right)^{\Upsilon} \ldots, \\
\left\langle W, \underline{\vec{E}}, \underline{\vec{B}}\left|\ldots \sqrt{V_{v}} \ldots\right| W, \underline{\vec{E}}, \underline{\vec{B}}\right\rangle \approx \ldots \ell_{P}^{3 / 2} \ldots,
\end{gathered}
$$

respectively. In our previous work [20] we have set $\Upsilon=0$ on the basis that the coarse graining approximation, defined by the scale $\mathcal{L}$, does not allow for the connection to be probed below $\frac{1}{\mathcal{L}}$. 
On the other hand, by adopting naive kinematical coherent states for representing semiclassical states, one would set $\Upsilon=1$ for two reasons: (i) to guarantee that (29) yields just zero in the limit $\hbar \rightarrow 0$, in agreement with a flat connection and (ii) because such an scaling would saturate the Heisenberg uncertainty relation [38]. Nonetheless, physical semiclassical states may imply a leading order contribution with $\Upsilon \neq 0,1$, thus we choose to consider $\Upsilon$ as a free parameter here.

In order to make the transition to the electric sector it is convenient to express the effective Hamiltonian $\mathrm{H}^{B}$ in terms of the magnetic field, which amounts to a redefinition of the expression (27) in the form

$$
\mathrm{H}^{B}=\int d^{3} x N(\vec{x}) \underline{B}_{r_{1}}(\vec{x}) \ldots \underline{B}_{r_{n}}(\vec{x})\left(\partial^{a_{1}} \ldots \partial^{a_{m}} \underline{B}_{r}(\vec{x})\right) R_{a_{1} \ldots a_{m}}{ }^{r r_{1} \ldots r_{n}}(\vec{x}) .
$$

The relation between the box-averaged tensors $R$ and $T$ is

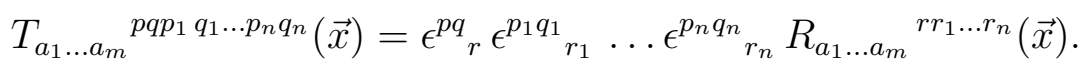

By expanding (22) to different powers in $s_{I}^{a}(\Delta)$ one can systematically determine all possible contributions to the effective electromagnetic Hamiltonian at a given order in $\ell_{P}$.

\section{The Calculation}

In this section we provide the details of the calculation of the Maxwell effective Hamiltonian up to order $\ell_{P}^{2}$. Let us start with the magnetic sector.

The two main ingredients in (22) which contribute to the expansion in powers of the segments $s_{I}(\Delta)$ are: (i) the trace factors involving the gravitational operators and (ii) the magnetic flux through each surface $F_{I J}(\Delta)$.

First we calculate the flux of the magnetic field through the surface $F_{I J}$. A convenient way to do this is via the Stokes theorem

$$
\Phi^{B}\left(F_{I J}\right)=\int_{F_{I J}} B_{a} n^{a} d^{2} x=\int_{F_{I J}}(\nabla \times \vec{A})_{a} n^{a} d^{2} x
$$




$$
\begin{aligned}
& =\int_{\alpha_{I J}} d t \dot{s}^{a}(t) A_{a}(t) \\
& =\int_{\vec{v}}^{\vec{v}+\vec{s}_{I}} \underline{A}_{a} d x^{a}+\int_{\vec{v}+\vec{s}_{I}}^{\vec{v}+\vec{s}_{J}} \underline{A}_{a} d x^{a}+\int_{\vec{v}+\vec{s}_{J}}^{\vec{v}} \underline{A}_{a} d x^{a} .
\end{aligned}
$$

Here the notation is $\vec{s}_{I}=\left\{s_{I}^{a}\right\}$ and analogously for $\vec{v}$. We are using straight line trajectories joining the vertices of the corresponding triangle.

The basic building block in (33) is

$$
\begin{aligned}
\int_{\vec{v}_{1}}^{\vec{v}_{2}} \underline{A}_{a}(\vec{x}) d x^{a} & =\int_{0}^{1} \underline{A}_{a}\left(\vec{v}_{1}+t\left(\vec{v}_{2}-\vec{v}_{1}\right)\right)\left(\vec{v}_{2}-\vec{v}_{1}\right)^{a} d t \\
& =\int_{0}^{1} \underline{A}_{a}\left(\vec{v}_{1}+t \vec{\Delta}\right) \Delta^{a} d t \\
& =\left(1+\frac{1}{2 !} \Delta^{b} \partial_{b}+\frac{1}{3 !}\left(\Delta^{b} \partial_{b}\right)^{2}+\ldots\right) \Delta^{a} A_{a}(v),
\end{aligned}
$$

with $\Delta^{a}=\left(\vec{v}_{2}-\vec{v}_{1}\right)^{a}$. The infinite series in parenthesis is

$$
F(x)=1+\frac{1}{2 !} x+\frac{1}{3 !} x^{2}+\frac{1}{4 !} x^{3}+\ldots=\frac{e^{x}-1}{x},
$$

yielding

$$
\int_{\vec{v}_{1}}^{\vec{v}_{2}} \underline{A}_{a}(\vec{x}) d x^{a}=F\left(\Delta^{a} \partial_{a}\right)\left(\Delta^{a} \underline{A}_{a}\left(\vec{v}_{1}\right)\right)
$$

In the following we employ the notation $\Delta^{a} V_{a}=\vec{\Delta} \cdot \vec{V}$. Using the above result in the three integrals appearing in (33) and after some algebra, we obtain

$$
\begin{aligned}
\Phi^{B}\left(F_{I J}\right) & =F_{1}\left(\vec{s}_{I} \cdot \nabla, \vec{s}_{J} \cdot \nabla\right) s_{J}^{a} s_{I}^{b}\left(\partial_{a} \underline{A}_{b}(\vec{v})-\partial_{b} \underline{A}_{a}(\vec{v})\right) \\
& =F_{1}\left(\vec{s}_{I} \cdot \nabla, \vec{s}_{J} \cdot \nabla\right) s_{J}^{a} s_{I}^{b} \epsilon_{a b c} B^{c}(v),
\end{aligned}
$$

where the gradient acts upon the coordinates of $\vec{v}$. The function $F_{1}$ is

$$
F_{1}(x, y)=\frac{y\left(\mathrm{e}^{x}-1\right)-x\left(\mathrm{e}^{y}-1\right)}{x y(y-x)}=-\sum_{n=1}^{\infty} \frac{1}{(n+1) !} \frac{x^{n}-y^{n}}{x-y} .
$$

Let us emphasize that $F_{1}(x, y)$ is just a power series in the variables $x$ and $y$. Expanding to fourth order in the segments $s_{I}^{a}$ we obtain

$$
\Phi^{B}\left(F_{I J}\right)=\left(1+\frac{1}{3}\left(s_{I}^{c}+s_{J}^{c}\right) \partial_{c}+\frac{1}{12}\left(s_{I}^{c} s_{I}^{d}+s_{I}^{c} s_{J}^{d}+s_{J}^{c} s_{J}^{d}\right) \partial_{c} \partial_{d}+\ldots\right) \times
$$




$$
\times \frac{1}{2} s_{I}^{a} s_{J}^{b} \epsilon_{a b c} B^{c}(v)
$$

Notice that the combination

$$
\frac{1}{2} s_{I}^{a} s_{J}^{b} \epsilon_{a b c}=\mathcal{A} n_{c}
$$

is just the oriented area of the triangle with vertex $v$ and sides $s_{I}^{c}, s_{J}^{c}$, joining at this vertex, having value $\mathcal{A}$ and unit normal vector $n_{c}$.

In order to make the bookkeeping clear, let us denote by $T$ the combination arising from (22) whose expectation value we are calculating

$$
T=-\frac{1}{2 Q^{2}} \frac{1}{\ell_{P}^{4}} \hat{w}_{i L \Delta} \hat{w}_{i P \Delta^{\prime}} \epsilon^{J K L} \epsilon^{M N P}\left(e^{-i \hat{\Phi}^{B}\left(F_{J K}\right)}-1\right)\left(e^{-i \hat{\Phi}^{B}\left(F_{M N}^{\prime}\right)}-1\right) .
$$

Here

$$
\hat{w}_{i L \Delta}=\operatorname{tr}\left(\tau_{i} h_{s_{L}(\Delta)}\left[h_{s_{L}(\Delta)}^{-1}, \sqrt{\hat{V}_{v}}\right]\right)
$$

Some remarks are in order before we proceed further. Our final goal is to obtain a power expansion of (41) up to order $\ell_{P}^{2}$. Since, as we will show in the sequel, the normalization factor converting magnetic operators inside the semiclassical expectation value into classical fields outside the expectation value is proportional to $\left(\ell_{P}\right)^{-1}$, we have to take some care regarding the expansion of the given quantities in powers of $s_{I}^{a}$. A detailed power counting analysis in the expression (41) shows that the term $\hat{w}_{i L(\Delta)}$ in (42) is to be expanded up to order $s^{3}$, while each magnetic factor $\left(e^{-i \hat{\Phi}^{B}\left(F_{J K}\right)}-1\right)$ is required to have the following properties: the terms proportional to $\underline{F}$ are to be expanded up to order $s^{4}$, those proportional to $\underline{F}^{2}$ up to order $s^{5}$ and finally those proportional to $\underline{F}^{3}$ up to order $s^{6}$. This will lead to the following contributions in $T$ : the terms proportional to $\underline{F}^{2}$ include the expansion up to order $s^{8}$, the terms proportional to $\underline{F}^{3}$ include the expansion up to order $s^{9}$ and the terms proportional to $\underline{F}^{4}$ include the expansion up to order $s^{10}$. The final result is that the semiclassical expectation value of the magnetic contribution $T$ will be proportional to $\ell_{P}^{3}$, which is incorporated in the volume element, times corrections up to order $\ell_{P}^{2}$. 
Let us now continue with the calculation of the contribution to (41) due to the magnetic flux by writing the expansion

$$
\begin{aligned}
\left(e^{-i \hat{\Phi}^{B}\left(F_{J K}(\Delta)\right)}-1\right)= & \sum_{n=1}^{\infty} \frac{(-i)^{n}}{n !}\left(\hat{\Phi}^{B}\left(F_{J K}\right)\right)^{n}=M_{1 J K(\Delta)}+M_{2 J K(\Delta)}+M_{3 J K(\Delta)} \\
& +\mathcal{O}\left(s^{7} \underline{F}^{3}\right)
\end{aligned}
$$

where

$$
\begin{aligned}
M_{1 J K(\Delta)}:= & s_{K}^{a} s_{J}^{b} \frac{i}{2 !} \underline{F}_{a b}, \\
M_{2 J K(\Delta)}:= & s_{K}^{a} s_{J}^{b} \frac{i}{3 !}\left(x_{J}+x_{K}\right) \underline{F}_{a b}-s_{K}^{a} s_{J}^{b} s_{K}^{c} s_{J}^{d} \frac{1}{8} \underline{F}_{a b} \underline{F}_{c d}, \\
M_{3 J K(\Delta):=} & s_{K}^{a} s_{J}^{b} \frac{i}{4 !}\left(x_{J}^{2}+x_{J} x_{K}+x_{K}^{2}\right) \underline{F}_{a b}-s_{K}^{a} s_{J}^{b} s_{K}^{c} s_{J}^{d}\left[\frac{1}{4 \cdot 3 !}\left(x_{J}+x_{K}\right) \underline{F}_{a b} \underline{F}_{c d}\right. \\
& \left.+\frac{1}{4 \cdot 3 !} \underline{F}_{a b}\left(x_{K}+x_{J}\right) \underline{F}_{c d}\right]-s_{K}^{a} s_{J}^{b} s_{K}^{c} s_{J}^{d} s_{K}^{e} s_{J}^{f} \frac{i}{4 \cdot 3 !} \underline{F}_{a b} \underline{F}_{c d} \underline{F}_{e f},
\end{aligned}
$$

according to the previous analysis. We are using the notation $x_{I}=\vec{s}_{I} \cdot \nabla=s_{I}^{a} \partial_{a}$. Let us remark that, contrary to the electric case, the magnetic contribution will incorporate non-linear terms due to the expansion of the exponential in powers of $\vec{B}$. This implies that the exact duality symmetry of Maxwell equations in vacuum will be lost due to quantum gravity corrections.

Next let us consider the gravitational contributions to (22), arising from (42), which we expand as

$$
\hat{w}_{i L \Delta}=s_{L}^{a} w_{i a}+s_{L}^{a} s_{L}^{b} w_{i a b}+s_{L}^{a} s_{L}^{b} s_{L}^{c} w_{i a b c}+\mathcal{O}\left(s^{4} w\right)
$$

with

$$
w_{i a}=\frac{1}{2}\left[A_{i a}, \sqrt{V}\right], \quad w_{i a b}=\frac{1}{8} \epsilon_{i j k}\left[A_{j a},\left[A_{k b}, \sqrt{V}\right]\right], \quad w_{i a b c}=-\frac{1}{48}\left[A_{j a},\left[A_{j b},\left[A_{i c}, \sqrt{V}\right]\right]\right] .
$$

The scaling properties of the above gravitational operators under the semiclassical expectation value are

$$
\left\langle W \underline{\underline{E}} \underline{\vec{B}}\left|\ldots w_{i a_{1} \ldots a_{n}} \ldots\right| W \underline{\vec{E}} \underline{\vec{B}}\right\rangle \rightarrow \frac{\ell_{P}^{3 / 2}}{\mathcal{L}^{n}}\left(\frac{\ell_{P}}{\mathcal{L}}\right)^{n \Upsilon}
$$


Let us emphasize that the result (47) is a consequence of the scaling of the expectation value of the connection given by (29).

For the product $\hat{w}_{i L \Delta} \hat{w}_{i P \Delta^{\prime}}$ we need only

$$
\hat{w}_{i L \Delta} \hat{w}_{i P \Delta^{\prime}}=U_{2 L P}+U_{3 L P}+U_{4 L P}+\mathcal{O}\left(s^{5} w^{2}\right)
$$

with

$$
\begin{aligned}
& U_{2 L P}=s_{L}^{a} s_{P}^{\prime d} w_{i a} w_{i d} \\
& U_{3 L P}=s_{L}^{a} s_{P}^{\prime d} s_{P}^{\prime e} w_{i a} w_{i d e}+s_{L}^{a} s_{L}^{b} s_{P}^{\prime d} w_{i a b} w_{i d}, \\
& U_{4 L P}=s_{L}^{a} s_{P}^{\prime d} s_{P}^{\prime} s_{P}^{\prime} w_{i a} w_{i d e f}+s_{L}^{a} s_{L}^{b} s_{P}^{\prime d} s_{P}^{\prime e} w_{i a b} w_{i d e}+s_{L}^{a} s_{L}^{b} s_{L}^{c} s_{P}^{\prime d} w_{i a b c} w_{i d}
\end{aligned}
$$

Here all the $w$ 's are evaluated at a common vertex $v$.

At this level it is convenient to state the result (no sum over $L$ )

$$
\begin{aligned}
s_{L}^{a} s_{L}^{b} w_{i a b} & =\frac{1}{8} s_{L}^{a} s_{L}^{b} \epsilon_{i j k}\left[A_{j a},\left[A_{k b}, \sqrt{V}\right]\right] \\
& =\frac{1}{8} s_{L}^{a} s_{L}^{b} \epsilon_{i j k}\left(A_{j a} A_{k b} \sqrt{V}-A_{j a} \sqrt{V} A_{k b}-A_{k b} \sqrt{V} A_{j a}+\sqrt{V} A_{k b} A_{j a}\right), \\
& =0,
\end{aligned}
$$

which holds due to symmetry properties. This leads to

$$
U_{3 L P}=0 .
$$

After taking the expectation value the terms contributing to order $\ell_{P}^{2}$ in (41) read

$$
\begin{gathered}
T=T_{0}+T_{1}+T_{2}+\mathcal{O}\left(\rightarrow \ell_{P}^{3}\right) \\
T_{0}=-\frac{1}{2 Q^{2}} \frac{1}{\ell_{P}^{4}} \epsilon^{J K L} \epsilon^{M N P}\left[U_{2 L P} M_{1 J K} M_{1 M N}^{\prime}\right], \\
T_{1}=-\frac{1}{2 Q^{2}} \frac{1}{\ell_{P}^{4}} \epsilon^{J K L} \epsilon^{M N P} U_{2 L P}\left[M_{1 J K} M_{2 M N}^{\prime}+M_{2 J K} M_{1 M N}^{\prime}\right], \\
T_{2}=-\frac{1}{2 Q^{2}} \frac{1}{\ell_{P}^{4}} \epsilon^{J K L} \epsilon^{M N P}\left[U_{2 L P}\left(M_{1 J K} M_{3 M N}^{\prime}+M_{3 J K} M_{1 M N}^{\prime}+M_{2 J K} M_{2 M N}^{\prime}\right)+\right. \\
\left.+U_{4 L P} M_{1 J K} M_{1 M N}^{\prime}\right] .
\end{gathered}
$$


Now we are ready to calculate the different contributions to the magnetic sector of the Hamiltonian (22), which we parameterize in terms of the tensor $\bar{W}_{a_{1} \ldots a_{m}}{ }^{r r_{1} \ldots r_{n}}$ introduced in (28).

Recalling that we are only interested in the pieces which are symmetric in the indices $r_{1}, r_{2}, \ldots, r_{n}$, the contribution $T_{0}$ produces

$$
\begin{aligned}
& R_{0}{ }^{r_{1} r_{2}}=\sum_{v \in \text { Box }} \frac{1}{2 Q^{2}}\left(\frac{2}{3 !} \frac{8}{E(v)}\right)^{2} \sum_{v(\Delta)=v\left(\Delta^{\prime}\right)=v} \frac{\mu^{2}}{4 \ell_{P}^{7}} \epsilon_{a b}^{r_{1}} \epsilon^{r_{2}}{ }_{u v} \epsilon^{J K L} \epsilon^{M N P} \times \\
& \times s_{K}^{a} s_{J}^{b} s_{L}^{c} s_{M}^{\prime v} s_{N}^{\prime u} s_{P}^{\prime d}\left\langle W \underline{\vec{E}} \underline{\vec{B}}\left|w_{i c} w_{i d}\right| W \underline{\vec{E}} \underline{\vec{B}}\right\rangle \text {. }
\end{aligned}
$$

In order to simplify the product of vectors $s_{L}^{a}\left(s^{\prime} M^{p}\right)$ appearing in the sequel and also to exhibit the internal symmetry properties of the quantities involved, it is convenient to keep in mind the relations

$$
\epsilon^{K J L} s_{K}^{a} s_{J}^{b} s_{L}^{c}=\operatorname{det}(s) \epsilon^{a b c}, \quad \operatorname{det}(s)=\operatorname{det}\left(s_{K}^{a}\right), \quad \epsilon^{a b p} \epsilon_{a b q}=2 \delta_{q}^{p} .
$$

In this way, (56) can be rewriten in the simpler form

$$
\begin{aligned}
R_{0}{ }^{r_{1} r_{2}}= & \sum_{v \in \operatorname{Box}} \frac{1}{2 Q^{2}}\left(\frac{2}{3 !} \frac{8}{E(v)}\right)^{2} \sum_{v(\Delta)=v\left(\Delta^{\prime}\right)=v} \frac{\mu^{2}}{\ell_{P}^{7}} \times \\
& \times \operatorname{det}(s) \operatorname{det}\left(s^{\prime}\right)\left\langle W, \underline{\vec{E}}, \underline{\vec{B}}\left|\frac{1}{2}\left\{w_{i}^{r_{1}}, w_{i}^{r_{2}}\right\}\right| W, \underline{\vec{E}}, \underline{\vec{B}}\right\rangle .
\end{aligned}
$$

The above equation implies

$$
R_{0}^{r_{1} r_{2}}=\frac{1}{2 Q^{2}} \mu_{P}^{2} \ell_{P}^{6} \frac{\ell_{P}^{3}}{\mathcal{L}^{2}}\left(\frac{\ell_{P}}{\mathcal{L}}\right)^{2 \Upsilon} \delta^{r_{1} r_{2}}=\frac{1}{2 Q^{2}} \delta^{r_{1} r_{2}},
$$

which reproduces the zeroth-order magnetic contribution (23) with the choice

$$
\mu=\left(\frac{\mathcal{L}}{\ell_{P}}\right)^{1+\Upsilon} .
$$


Now let us consider the correction arising from $T_{1}$, which leads to the following contribution in the effective Hamiltonian

$$
\begin{array}{r}
\mathrm{H}_{11}^{B}=\sum_{\operatorname{Box}(\vec{x})} \sum_{v \in \operatorname{Box}} \frac{1}{2 Q^{2}}\left(\frac{2}{3 !} \frac{8}{E(v)}\right)^{2} \ell_{P}^{3} \sum_{v(\Delta)=v\left(\Delta^{\prime}\right)=v}-\frac{1}{\ell_{P}^{7}} \epsilon^{J K L} \epsilon^{M N P} s_{L}^{c} s_{P}^{\prime d} s_{K}^{a} s_{J}^{b} \times \\
\times\langle W, \underline{\vec{E}}, \underline{\vec{B}}|\left\{w_{i c}, w_{i d}\right\} \frac{i}{2} \underline{F}_{a b}\left(s^{\prime v} s^{\prime q}{ }_{M} \frac{i}{3 !}\left(x^{\prime}{ }_{M}+x^{\prime}{ }_{N}\right) \underline{F}_{v q}-\right. \\
\left.-s^{\prime \prime}{ }_{N} s^{\prime q}{ }_{M}{ }^{\prime \prime}{ }_{N}{ }^{\prime} s_{M}^{\prime t} \frac{1}{8} \underline{F}_{v q} \underline{F}_{r t}\right)|W, \underline{\vec{E}}, \underline{\vec{B}}\rangle .
\end{array}
$$

In the above expression we have interchanged the summations over $\Delta$ and $\Delta^{\prime}$ in order to rewrite $w_{i c} w_{i d}$ as $\frac{1}{2}\left\{w_{i c}, w_{i d}\right\}$. We further separate the above contribution to $\mathrm{H}_{11}^{B}$ in two parts: (i) the first contains two powers in the magnetic field and leads to $R_{11 a_{1}}{ }^{r r_{1}}$ and (ii) the second one contains three powers in the magnetic field and leads to the completely symmetric tensor $R_{11} r_{1} r_{2} r_{3}$. Since we have no symmetric tensor with three indices at our disposal, the latter contribution is zero. Thus we concentrate in the first one

$$
\begin{aligned}
R_{11}{ }^{a_{1} r r_{1}}= & \sum_{v \in \operatorname{Box}} \frac{1}{2 Q^{2}}\left(\frac{2}{3 !} \frac{8}{E(v)}\right)^{2} \sum_{v(\Delta)=v\left(\Delta^{\prime}\right)=v} \frac{\mu^{2}}{6 \ell_{P}^{7}} \epsilon^{J K L} \epsilon^{M N P} s_{L}^{c} s_{P}^{\prime d} \times \\
& s_{K}^{a} s_{J}^{b} s_{N}^{\prime v} s_{M}^{\prime q}{ }_{M}^{\prime a_{1}} \epsilon^{r_{1}}{ }_{a b} \epsilon_{v q}^{r}\left\langle W, \underline{\vec{E}}, \underline{\vec{B}}\left|\left\{w_{i c}, w_{i d}\right\}\right| W, \underline{\vec{E}}, \underline{\vec{B}}\right\rangle .
\end{aligned}
$$

In this case the internal symmetry properties are hard to make explicit. In order to determine whether or not the above contribution is zero we contract (62) with the only three index tensor at our disposal: $\epsilon^{a_{1} r r_{1}}$. The result is

$$
\begin{aligned}
\epsilon_{a_{1} r r_{1}} R_{11} a_{1} r r_{1} & =-\sum_{v \in \operatorname{Box}} \frac{1}{2 Q^{2}}\left(\frac{2}{3 !} \frac{8}{E(v)}\right)^{2} \sum_{v(\Delta)=v\left(\Delta^{\prime}\right)=v} \frac{\mu^{2}}{6 \ell_{P}^{7}} \operatorname{det}(s) \epsilon^{M N P} \\
& \times\left\langle W, \underline{\vec{E}}, \underline{\vec{B}}\left|\left\{w_{i c}, w_{i d}\right\}\right| W, \underline{\vec{E}}, \underline{\vec{B}}\right\rangle\left(s_{P}^{\prime d} s^{\prime c}{ }_{M} s^{\prime q}{ }_{N}^{\prime} s_{q M}+s_{P}^{\prime d} s^{\prime c}{ }_{N} s^{\prime q}{ }_{M} s^{\prime}{ }_{q M}\right) .
\end{aligned}
$$

Inside the expectation value, upper spatial indices have been lowered by the flat metric. By symmetry requirements, the second term in parenthesis in (63) yields zero. Nevertheless, the 
first one gives the result

$$
R_{11}^{a_{1} r r_{1}}=\kappa_{8} \frac{\mu^{2}}{Q^{2} \ell_{P}^{7}} \ell_{P}^{7} \frac{\ell_{P}^{3}}{\mathcal{L}^{2}}\left(\frac{\ell_{P}}{\mathcal{L}}\right)^{2 \Upsilon} \epsilon^{a_{1} r r_{1}},
$$

which produces a parity-violating term in the magnetic sector of the effective Hamiltonian.

The next contribution arises from $T_{2}$ and can be separated into three pieces

$$
\begin{aligned}
\mathrm{H}_{21}^{B}= & \sum_{v \in V(\gamma)} \frac{1}{2 Q^{2}}\left(\frac{2}{3 !} \frac{8}{E(v)}\right)^{2} \ell_{P}^{3} \sum_{v(\Delta)=v\left(\Delta^{\prime}\right)=v}-\frac{1}{\ell_{P}^{7}} \epsilon^{J K L} \epsilon^{M N P} \times \\
& \times\left\langle W, \underline{\vec{E}}, \underline{\vec{B}}\left|U_{2 L P}\left(M_{1 J K} M_{3 M N}^{\prime}+M_{3 J K} M_{1 M N}^{\prime}\right)\right| W, \underline{\vec{E}}, \underline{\vec{B}}\right\rangle, \\
\mathrm{H}_{22}^{B}= & \sum_{v \in V(\gamma)} \frac{1}{2 Q^{2}}\left(\frac{2}{3 !} \frac{8}{E(v)}\right)^{2} \ell_{P}^{3} \sum_{v(\Delta)=v\left(\Delta^{\prime}\right)=v}-\frac{1}{\ell_{P}^{7}} \epsilon^{J K L} \epsilon^{M N P} \times \\
& \times\left\langle W, \underline{\vec{E}} \underline{\vec{B}}\left|U_{2 L P} M_{2 J K} M_{2 M N}^{\prime}\right| W, \underline{\vec{E}}, \underline{\vec{B}}\right\rangle, \\
\mathrm{H}_{23}^{B}= & \sum_{v \in V(\gamma)} \frac{1}{2 Q^{2}}\left(\frac{2}{3 !} \frac{8}{E(v)}\right)^{2} \ell_{P}^{3} \sum_{v(\Delta)=v\left(\Delta^{\prime}\right)=v}-\frac{1}{\ell_{P}^{7}} \epsilon^{J K L} \epsilon^{M N P} \times \\
& \times\left\langle W, \underline{\vec{E}}, \underline{\vec{B}}\left|U_{4 L P} M_{1 J K} M_{1 M N}^{\prime}\right| W, \underline{\vec{E}}, \underline{\vec{B}}\right\rangle .
\end{aligned}
$$

Let us start discussing $\mathrm{H}_{21}^{B}$. After some algebra we obtain

$$
\begin{aligned}
\mathrm{H}_{21}^{B}=\sum_{v \in V(\gamma)} \frac{1}{2 Q^{2}} & \left(\frac{2}{3 !} \frac{8}{E(v)}\right)^{2} \ell_{P}^{3} \sum_{v(\Delta)=v\left(\Delta^{\prime}\right)=v}-\frac{i}{\ell_{P}^{7}} \epsilon^{J K L} \epsilon^{M N P} \times \\
& \times s_{L}^{a} s_{P}^{\prime d} s_{N}^{\prime x} s_{M}^{\prime q}\langle W, \underline{\vec{E}}, \underline{\vec{B}}| w_{i a} w_{i d}\left(s_{K}^{r} s_{J}^{t} \frac{i}{4 !}\left(x_{J}^{2}+x_{J} x_{K}+x_{K}^{2}\right) \underline{F}_{r t}\right. \\
& -s_{K}^{r} s_{J}^{t} s_{K}^{u} s_{J}^{v}\left[\frac{1}{4 \cdot 3 !}\left(x_{J}+x_{K}\right) \underline{F}_{r t} \underline{F}_{u v}+\frac{1}{4 \cdot 3 !} \underline{F}_{r t}\left(x_{K}+x_{J}\right) \underline{F}_{u v}\right] \\
& \left.-s_{K}^{r} s_{J}^{t} s_{K}^{u} s_{J}^{v} s_{K}^{w} s_{J}^{z} \frac{i}{4 \cdot 3 !} \underline{F}_{r t} \underline{F}_{u v} \underline{F}_{w z}\right) \underline{F}_{x q}|W, \underline{\vec{E}}, \underline{\vec{B}}\rangle,
\end{aligned}
$$

which naturally splits into the following pieces

$$
\mathrm{H}_{211}^{B}=\sum_{v \in V(\gamma)} \frac{1}{2 Q^{2}}\left(\frac{2}{3 !} \frac{8}{E(v)}\right)^{2} \ell_{P}^{3} \sum_{v(\Delta)=v\left(\Delta^{\prime}\right)=v}-\frac{i}{\ell_{P}^{7}} \epsilon^{J K L} \epsilon^{M N P} \times
$$




$$
\begin{aligned}
& \times s_{L}^{a} s_{P}^{\prime d} s_{N}^{\prime x} s_{M}^{\prime q}\left\langle W, \underline{\vec{E}}, \underline{\vec{B}}\left|w_{i a} w_{i d}\left(s_{K}^{r} s_{J}^{t} \frac{i}{4 !}\left(x_{J}^{2}+x_{J} x_{K}+x_{K}^{2}\right) \underline{F}_{r t}\right) \underline{F}_{x q}\right| W, \underline{\vec{E}}, \underline{\vec{B}}\right\rangle \\
& \mathrm{H}_{212}^{B}= \sum_{v \in V(\gamma)} \frac{1}{2 Q^{2}}\left(\frac{2}{3 !} \frac{8}{E(v)}\right)^{2} \ell_{P}^{3} \sum_{v(\Delta)=v\left(\Delta^{\prime}\right)=v} \frac{i}{\ell_{P}^{7}} \epsilon^{J K L} \epsilon^{M N P} \times \\
& \times s_{L}^{a} s_{P}^{\prime d} s_{N}^{\prime x} s_{M}^{\prime q}\langle W, \underline{\vec{E}}, \underline{\vec{B}}| w_{i a} w_{i d}\left(s _ { K } ^ { r } s _ { J } ^ { t } s _ { K } ^ { u } s _ { J } ^ { v } \left[\frac{1}{4 \cdot 3 !}\left(x_{J}+x_{K}\right) \underline{F}_{r t} \underline{F}_{u v}\right.\right. \\
&\left.\left.+\frac{1}{4 \cdot 3 !} \underline{F}_{r t}\left(x_{K}+x_{J}\right) \underline{F}_{u v}\right]\right) \underline{F}_{x q}|W, \underline{\vec{E}}, \underline{\vec{B}}\rangle, \\
& \mathrm{H}_{213}^{B}= \sum_{v \in V(\gamma)} \frac{1}{2 Q^{2}}\left(\frac{2}{3 !} \frac{8}{E(v)}\right)^{2} \ell_{P}^{3} \sum_{v(\Delta)=v\left(\Delta^{\prime}\right)=v} \frac{i}{\ell_{P}^{7}} \epsilon^{J K L} \epsilon^{M N P} \times \\
& \quad \times s_{L}^{a} s_{P}^{\prime d} s_{N}^{\prime x} s_{M}^{\prime q}\left\langle W, \underline{\vec{E}} \underline{\vec{B}}\left|w_{i a} w_{i d}\left(s_{K}^{r} s_{J}^{t} s_{K}^{u} s_{J}^{v} s_{K}^{w} s_{J}^{z} \frac{i}{4 \cdot 3 !} \underline{F}_{r t} \underline{F}_{u v} \underline{F}_{w z}\right) \underline{F}_{x q}\right| W, \underline{\vec{E}} \underline{\vec{B}}\right\rangle .
\end{aligned}
$$

Next we calculate the corresponding $R$ tensors. The first one is

$$
\begin{aligned}
R_{211} a_{1} a_{2} r r_{1}= & \sum_{v \in \operatorname{Box}} \frac{1}{2}\left(\frac{2}{3 !} \frac{8}{E(v)}\right)^{2} \sum_{v(\Delta)=v\left(\Delta^{\prime}\right)=v} \frac{2 \mu^{2}}{4 ! Q^{2} \ell_{P}^{7}} \operatorname{det}\left(s^{\prime}\right) \operatorname{det}(s) \\
& \epsilon_{N K J}\left(s^{-1}\right)^{r N} \epsilon^{J K L} s_{L}^{a}\left(2 s_{J}^{a_{1}} s_{J}^{a_{2}}+s_{J}^{a_{1}} s_{K}^{a_{2}}\right)\left\langle W, \underline{\vec{E}}, \underline{\vec{B}}\left|w_{i a} w_{i}^{r_{1}}\right| W, \underline{\vec{E}}, \underline{\vec{B}}\right\rangle, \\
R_{211}^{a_{1} a_{2} r r_{1}}= & \frac{\mu^{2}}{Q^{2} \ell_{P}^{7}} \ell_{P}^{8} \frac{\ell_{P}^{3}}{\mathcal{L}^{2}}\left(\frac{\ell_{P}}{\mathcal{L}}\right)^{2 \Upsilon}\left(\kappa_{6} \delta^{a_{1} a_{2}} \delta^{r r_{1}}+\kappa_{7}\left(\delta^{a_{1} r} \delta^{a_{2} r_{1}}+\delta^{a_{2} r} \delta^{a_{1} r_{1}}\right)\right),
\end{aligned}
$$

that we have constructed using the most general tensor with four indices, which is symmetrical in $a_{1}$ and $a_{2}$. The terms proportional to $\kappa_{7}$ contribute with $\underline{B}_{a} \partial^{a} \partial^{b} \underline{B}_{b}$ to the effective Hamiltonian. Keeping this term will be useful to construct the electric sector later. Notice that we have also introduced the inverse matrix $\left(s^{-1}\right)_{m}^{N}$ such that

$$
\left(s^{-1}\right)_{m}^{P} s_{Q}^{m}=\delta_{Q}^{P}, \quad s_{M}^{a}\left(s^{-1}\right)_{b}^{M}=\delta_{b}^{a},
$$

which elements scale as $\ell_{P}^{-1}$. 
Our next contribution, arising from (70), is zero because the object (no sum over $J$ and $K$ )

$$
s_{K}^{r} s_{J}^{t} s_{K}^{u} s_{J}^{v} \underline{F}_{u v}\left(x_{J}+x_{K}\right) \underline{F}_{r t},
$$

which is symmetrical in $J K$, appears contracted with $\epsilon^{J K L}$.

Now we consider

$$
\begin{aligned}
\mathrm{H}_{213}^{B}= & \sum_{\operatorname{Box}(\vec{x})} \underline{B}^{b}(\vec{x}) \underline{B}^{c}(\vec{x}) \underline{B}^{f}(\vec{x}) \underline{B}^{e}(\vec{x}) \sum_{v \in B o x} \frac{1}{2 Q^{2}}\left(\frac{2}{3 !} \frac{8}{E(v)}\right)^{2} \ell_{P}^{3} \sum_{v(\Delta)=v\left(\Delta^{\prime}\right)=v}-\frac{\mu^{4}}{4 \times 3 ! \ell_{P}^{7}} \\
& 2 \operatorname{det}\left(s^{\prime}\right)(\operatorname{det}(s))^{3} \epsilon^{J K L} s_{L}^{a}\left(\epsilon_{U K J}\left(s^{-1}\right)_{b}^{U}\right)\left(\epsilon_{V K J}\left(s^{-1}\right)_{c}^{V}\right)\left(\epsilon_{X K J}\left(s^{-1}\right)_{f}^{X}\right) \times \\
& \times\left\langle W, \underline{\vec{E}}, \underline{\vec{B}}\left|w_{i a} w_{i e}\right| W, \underline{\vec{E}}, \underline{\vec{B}}\right\rangle
\end{aligned}
$$

where we have introduced the relation

$$
\epsilon_{m b c} s_{J}^{b} s_{L}^{c}=\operatorname{det}(s) \epsilon_{K J L}\left(s^{-1}\right)_{m}^{K},
$$

obtained from (57). The further properties

$$
\epsilon^{L K J} \epsilon_{U K J} \epsilon_{V K J} \epsilon_{X K J} \Longrightarrow L=U=V=X, \quad \epsilon^{1 K J} \epsilon_{1 K J} \epsilon_{1 K J} \epsilon_{1 K J}=2 \epsilon^{123} \epsilon_{123} \epsilon_{123} \epsilon_{123}=-2,
$$

allow us to rewrite the corresponding tensor in the simpler form

$$
\begin{aligned}
R_{213 a_{1} a_{2} a_{3} a_{4}}= & \sum_{v \in \operatorname{Box}} \frac{1}{2 Q^{2}}\left(\frac{2}{3 !} \frac{8}{E(v)}\right)^{2} \sum_{v(\Delta)=v\left(\Delta^{\prime}\right)=v}-\frac{\mu^{4}}{3 ! \ell_{P}^{7}} \operatorname{det}\left(s^{\prime}\right)(\operatorname{det}(s))^{3} \times \\
& \times s_{L}^{a}\left(s^{-1}\right)_{a_{1}}^{L}\left(s^{-1}\right)_{a_{2}}^{L}\left(s^{-1}\right)_{a_{3}}^{L}\left\langle W, \underline{\vec{E}}, \underline{\vec{B}}\left|w_{i a} w_{i a_{4}}\right| W, \underline{\vec{E}}, \underline{\vec{B}}\right\rangle .
\end{aligned}
$$

This shows explicitly the symmetry in the indices $a_{1}, a_{2}, a_{3}$ and leads to

$$
R_{213 a_{1} a_{2} a_{3} a_{4}}=\frac{\mu^{4}}{Q^{2} \ell_{P}^{7}} \ell_{P}^{10} \frac{\ell_{P}^{3}}{\mathcal{L}^{2}}\left(\frac{\ell_{P}}{\mathcal{L}}\right)^{2 \Upsilon} \kappa_{4}\left(\delta_{a_{1} a_{2}} \delta_{a_{3} a_{4}}+\delta_{a_{1} a_{3}} \delta_{a_{2} a_{4}}+\delta_{a_{1} a_{4}} \delta_{a_{2} a_{3}}\right),
$$

where we have written the most general four index tensor at our disposal, which is completely symmetric in three indices. This implies the complete symmetry in the four indices. The correction (79) leads to cubic non-linear terms in the equation of motion. 
Now we continue with the correction arising from (66), which reduces to

$$
\begin{aligned}
\mathrm{H}_{22}^{B}= & \sum_{v \in \operatorname{Box}} \frac{1}{2 Q^{2}}\left(\frac{2}{3 !} \frac{8}{E(v)}\right)^{2} \ell_{P}^{3} \sum_{v(\Delta)=v\left(\Delta^{\prime}\right)=v} \frac{4}{(3 !)^{2} \ell_{P}^{7}} \epsilon^{J K L} \epsilon^{M N P} \times \\
& \times s_{L}^{a} s_{P}^{\prime d}\left\langle W, \underline{\vec{E}}, \underline{\vec{B}}\left|w_{i a} w_{i d}\left(s_{K}^{u} s_{J}^{y} x_{J} \underline{F}_{u y}\right)\left(s_{N}^{\prime r} s_{M}^{\prime s} x_{M}^{\prime} \underline{F}_{r s}\right)\right| W, \underline{\vec{E}}, \underline{\vec{B}}\right\rangle,
\end{aligned}
$$

because the terms quadratic in $\underline{F}$ are symmetric in $J, K$ and $M, N$ respectively.

¿From the above we read

$$
\begin{aligned}
R_{22}{ }^{a_{1} a_{2} r r_{1}}= & \sum_{v \in \operatorname{Box}} \frac{1}{2 Q^{2}}\left(\frac{2}{3 !} \frac{8}{E(v)}\right)^{2} \sum_{v(\Delta)=v\left(\Delta^{\prime}\right)=v} \frac{4 \mu^{2}}{(3 !)^{2} \ell_{P}^{7}} \operatorname{det}(s) \operatorname{det}\left(s^{\prime}\right) \times \\
& \times\left(-\epsilon_{Y M N} \epsilon^{P M N} s_{P}^{\prime d} s_{M}^{\prime a_{2}}\right)\left(\epsilon_{X J K} \epsilon^{L J K} s_{L}^{a} s_{J}^{a_{1}}\right)\left(s^{-1}\right)^{X r_{1}}\left(s^{\prime-1}\right)^{Y r} \\
& \times\left\langle W, \underline{\vec{E}}, \underline{\vec{B}}\left|w_{i a} w_{i d}\right| W, \underline{\vec{E}}, \underline{\vec{B}}\right\rangle .
\end{aligned}
$$

Here the internal symmetry properties are rather obscure. Nevertheless, the symmetry properties induced by the classical magnetic field factors imply that the above tensor must be proportional to the most general tensor with four indices which is symmetric in $a_{1} a_{2}$. Then we have

$$
R_{22}{ }^{a_{1} a_{2} r r_{1}}=\frac{\mu^{2}}{Q^{2} \ell_{P}^{7}} \ell_{P}^{8} \frac{\ell_{P}^{3}}{\mathcal{L}^{2}}\left(\frac{\ell_{P}}{\mathcal{L}}\right)^{2 \Upsilon}\left(\kappa_{9} \delta^{a_{1} a_{2}} \delta^{r r_{1}}+\kappa_{10}\left(\delta^{a_{1} r} \delta^{a_{2} r_{1}}+\delta^{a_{2} r} \delta^{a_{1} r_{1}}\right)\right) .
$$

This contribution is of the same kind as the one given by $R_{211}{ }^{a_{1} a_{2} r r_{1}}$.

Finally we are left with

$$
\begin{aligned}
\mathrm{H}_{23}^{B}= & \sum_{\operatorname{Box}(\vec{x})} \underline{B}_{r_{1}}(\vec{x}) \underline{B}_{r}(\vec{x}) \sum_{v \in \operatorname{Box}(\vec{x})} \frac{1}{2 Q^{2}}\left(\frac{2}{3 !} \frac{8}{E(v)}\right)^{2} \ell_{P}^{3} \sum_{v(\Delta)=v\left(\Delta^{\prime}\right)=v} \frac{\mu^{2}}{4 \ell_{P}^{7}} \times \\
& \times \epsilon^{J K L} \epsilon^{M N P} s_{K}^{u} s_{J}^{v} s_{N}^{\prime s} s_{M}^{\prime y}\left(s_{L}^{d} s_{P}^{\prime a} s_{P}^{\prime b} s_{P}^{\prime c}\right) \epsilon_{u v}{ }^{r} \epsilon_{x y}\left\langle W, \underline{\vec{E}}, \underline{\vec{B}}\left|\left\{w_{i d}, w_{i a b c}\right\}\right| W, \underline{\vec{E}}, \underline{\vec{B}}\right\rangle,
\end{aligned}
$$

which leads to

$$
\begin{aligned}
{R_{23}{ }^{r r_{1}}=} & \sum_{v \in \operatorname{Box}(\vec{x})} \frac{1}{2 Q^{2}}\left(\frac{2}{3 !} \frac{8}{E(v)}\right)^{2} \sum_{v(\Delta)=v\left(\Delta^{\prime}\right)=v}-\frac{\mu^{2}}{\ell_{P}^{7}} \operatorname{det}\left(s^{\prime}\right) \operatorname{det}(s) \\
& \times\left(s^{\prime-1}\right)^{r P}\left(s_{P}^{\prime a} s_{P}^{\prime b} s_{P}^{\prime c}\right)\left\langle W, \underline{\vec{E}}, \underline{\vec{B}}\left|\left\{w_{i}^{r_{1}}, w_{i a b c}\right\}\right| W, \underline{\vec{E}}, \underline{\vec{B}}\right\rangle .
\end{aligned}
$$


Taking the symmetric part, we have

$$
R_{23}{ }^{r r_{1}}=\kappa_{11} \frac{\mu^{2}}{Q^{2} \ell_{P}^{7}} \ell_{P}^{8} \frac{\ell_{P}^{3}}{\mathcal{L}^{4}}\left(\frac{\ell_{P}}{\mathcal{L}}\right)^{4 \Upsilon} \delta^{r r_{1}} .
$$

Adding all previous contributions, we obtain the magnetic sector of the effective Hamiltonian, up to order $\ell_{P}^{2}$,

$$
\begin{aligned}
\mathrm{H}^{B}=\frac{1}{Q^{2}} \int d^{3}(\vec{x})[ & \left(1+\theta_{7}\left(\frac{\ell_{P}}{\mathcal{L}}\right)^{2+2 \Upsilon}\right) \frac{1}{2} \underline{\vec{B}}^{2}+\ell_{P}^{2}\left(\theta_{2} \underline{B}^{a} \partial_{a} \partial_{b} \underline{B}^{b}+\theta_{3} \underline{B}^{a} \nabla^{2} \underline{B}_{a}\right)+ \\
& \left.+\theta_{8} \ell_{P} \underline{\vec{B}} \cdot(\nabla \times \underline{\vec{B}})+\theta_{4} \mathcal{L}^{2} \ell_{P}^{2}\left(\frac{\mathcal{L}}{\ell_{P}}\right)^{2 \Upsilon}\left(\underline{\vec{B}}^{2}\right)^{2}+\ldots\right] .
\end{aligned}
$$

The numbers $\theta_{i}$ are linear combinations of the corresponding $\kappa_{j}$ appearing in the tensors $R$. The correspondences are

$$
\kappa_{7}, \kappa_{10} \rightarrow \theta_{2}, \quad \kappa_{6}, \kappa_{9} \rightarrow \theta_{3}, \quad \kappa_{4} \rightarrow \theta_{4}, \quad \kappa_{11} \rightarrow \theta_{7}, \quad, \kappa_{8} \rightarrow \theta_{8}
$$

As it is pointed out after (22), the electric sector of the effective Hamiltonian can be obtained by changing $\vec{B}$ into $\vec{E}$ in the quadratic contribution of the corresponding magnetic sector. In this way the complete electromagnetic effective Hamiltonian becomes

$$
\begin{aligned}
\mathrm{H}^{E M}=\frac{1}{Q^{2}} \int d^{3} \vec{x}[(1 & \left.+\theta_{7}\left(\frac{\ell_{P}}{\mathcal{L}}\right)^{2+2 \Upsilon}\right) \frac{1}{2}\left(\underline{\vec{B}}^{2}+\underline{\vec{E}}^{2}\right)+\theta_{3} \ell_{P}^{2}\left(\underline{B}^{a} \nabla^{2} \underline{B}_{a}+\underline{E}^{a} \nabla^{2} \underline{E}_{a}\right)+ \\
& +\theta_{2} \ell_{P}^{2} \underline{\underline{E}}^{a} \partial_{a} \partial_{b} \underline{E}^{b}+\theta_{8} \ell_{P}(\underline{\vec{B}} \cdot(\nabla \times \underline{\vec{B}})+\underline{\vec{E}} \cdot(\nabla \times \underline{\vec{E}})) \\
& \left.+\theta_{4} \mathcal{L}^{2} \ell_{P}^{2}\left(\frac{\mathcal{L}}{\ell_{P}}\right)^{2 \Upsilon}\left(\underline{\vec{B}}^{2}\right)^{2}+\ldots\right]
\end{aligned}
$$

up to order $\ell_{P}^{2}$. 


\section{Modified Maxwell equations and dispersion relations in vacuum}

Since no confusion arises in the sequel, we eliminate the underline in all electromagnetic quantities. From the effective Hamiltonian (88) we obtain the equations of motion

$$
\begin{array}{r}
A(\nabla \times \vec{B})-\frac{1}{c} \frac{\partial \vec{E}}{\partial t}+2 \ell_{P}^{2} \theta_{3} \nabla^{2}(\nabla \times \vec{B})-2 \theta_{8} \ell_{P} \nabla^{2} \vec{B}+4 \theta_{4} \mathcal{L}^{2}\left(\frac{\mathcal{L}}{\ell_{P}}\right)^{2 \Upsilon} \ell_{P}^{2} \nabla \times\left(\vec{B}^{2} \vec{B}\right)=0 \\
A(\nabla \times \vec{E})+\frac{1}{c} \frac{\partial \vec{B}}{\partial t}+2 \ell_{P}^{2} \theta_{3} \nabla^{2}(\nabla \times \vec{E})-2 \theta_{8} \ell_{P} \nabla^{2} \vec{E}=0
\end{array}
$$

where

$$
A=1+\theta_{7}\left(\frac{\ell_{P}}{\mathcal{L}}\right)^{2+2 \Upsilon}
$$

The above equations are supplemented by the condition $\nabla \cdot \vec{B}=0$, together with the constraint $\nabla \cdot \vec{E}=0$, appropriate for vacuum.

Next we calculate the dispersion relations arising from the modified Maxwell equations (89) and (90). Neglecting the non-linear part and introducing the plane wave ansatz

$$
\vec{E}=\vec{E}_{0} e^{i(\vec{k} \vec{x}-\omega t)}, \quad \vec{B}=\vec{B}_{0} e^{i(\vec{k} \vec{x}-\omega t)}, \quad k=|\vec{k}|,
$$

we get

$$
\begin{aligned}
\vec{E}_{0} \cdot \vec{k}=0, \quad \vec{B}_{0} \cdot \vec{k} & =0, \\
\left(\vec{k} \times \vec{E}_{0}\right)\left[A-2 \theta_{3}\left(\ell_{P} k\right)^{2}\right]-2 i \theta_{8} \ell_{P} \quad k^{2} \vec{E}_{0}-\frac{\omega}{c} \vec{B}_{0} & =0, \\
\left(\vec{k} \times \vec{B}_{0}\right)\left[A-2 \theta_{3}\left(\ell_{P}\right.\right. & \left.k)^{2}\right]-2 i \theta_{8} \ell_{P} \\
k^{2} \vec{B}_{0}+\frac{\omega}{c} \vec{E}_{0} & =0,
\end{aligned}
$$

which imply the following dispersion relations

$$
\omega=c k\left(1+\theta_{7}\left(\frac{\ell_{P}}{\mathcal{L}}\right)^{2+2 \Upsilon}-2 \theta_{3}\left(k \ell_{P}\right)^{2} \pm 2 \theta_{8}\left(k \ell_{P}\right)\right)
$$


The \pm signs correspond to the different polarizations of the photon. From the above we obtain the speed of the photon

$$
\frac{v}{c}=\left.\frac{1}{c} \frac{d \omega}{d k}\right|_{\mathcal{L}=1 / k}=1 \pm 4 \theta_{8}\left(k \ell_{P}\right)-6 \theta_{3}\left(k \ell_{P}\right)^{2}+\theta_{7}\left(k \ell_{P}\right)^{2+2 \Upsilon}+\ldots
$$

The last expression gives $v$ expanded to leading order in $\ell_{P}$ where we have estimated $\mathcal{L}$ as $1 / k$, which is its maximal value. Clearly (97) is valid only for momenta satisfying $\left(\ell_{P} k\right)<<1$.

To first order in $\left(k \ell_{P}\right)$ we have only the helicity dependent correction found already by Gambini and Pullin [9]. As far as the $\Upsilon$ dependent terms we have either a quadratic $(\Upsilon=0)$ or a quartic $(\Upsilon=1)$ correction. The only possibility to have a first order helicity independent correction amounts to set $\Upsilon=-1 / 2$ which corresponds to that of Ellis et. al. [10. However, we do not have an interpretation for such a value of $\Upsilon$.

\section{$7 \quad$ Effect of non-linear terms}

In this section we explore some implications of the non-linear term in the Maxwell equations induced by the quantum gravity corrections to order $\ell_{P}^{2}$.

Following reference [39 we study the propagation of waves in the presence of a constant magnetic field $\vec{B}_{0}$. To do this, let us write

$$
\vec{B}=\vec{B}_{0}+\vec{b}
$$

and consider only the contribution of the non-linear term in the effective Hamiltonian. Namely, let us set $\theta_{3}=\theta_{7}=\theta_{8}=0$. After linearizing in $\vec{b}$, the field equations reduce to

$$
\begin{array}{r}
\nabla \times \vec{b}-\frac{1}{c} \frac{\partial \vec{E}}{\partial t}+4 \bar{\theta}_{4} \mathcal{L}^{2} \ell_{P}^{2} \nabla \times\left(\vec{B}_{0}^{2} \vec{b}+2\left(\vec{B}_{0} \cdot \vec{b}\right) \vec{B}_{0}\right)=0 \\
\nabla \cdot \vec{E}=0, \quad \nabla \cdot \vec{b}=0, \quad \nabla \times \vec{E}+\frac{1}{c} \frac{\partial \vec{b}}{\partial t}=0,
\end{array}
$$


with $\bar{\theta}_{4}=\left(\frac{\mathcal{L}}{\ell_{P}}\right)^{2 \Upsilon} \theta_{4}$. Now we look for plane wave solutions of (99) with

$$
\vec{E}=\vec{E}_{0} e^{i(\vec{k} \vec{x}-\omega t)}, \quad \vec{b}=\vec{b}_{0} e^{i(\vec{k} \vec{x}-\omega t)},
$$

obtaining the conditions

$$
\begin{array}{r}
\vec{E}_{0} \cdot \vec{k}=0, \quad \vec{b}_{0} \cdot \vec{k}=0, \quad\left(\vec{k} \times \vec{E}_{0}\right)-\frac{\omega}{c} \vec{b}_{0}=0, \\
\left(1+4 \bar{\theta}_{4} \mathcal{L}^{2} \ell_{P}^{2} \vec{B}_{0}^{2}\right)\left(\vec{k} \times \vec{b}_{0}\right)+\frac{\omega}{c} \vec{E}_{0}+8 \bar{\theta}_{4} \mathcal{L}^{2} \ell_{P}^{2}\left(\vec{b}_{0} \cdot \vec{B}_{0}\right)\left(\vec{k} \times \vec{B}_{0}\right)=0 .
\end{array}
$$

Substituting in (102) the expression for $\vec{b}_{0}$ obtained from the third equation in (101) we are left with

$$
\left.\left(\frac{\omega^{2}}{c^{2}}-k^{2}\left(1+4 \bar{\theta}_{4} \mathcal{L}^{2} \ell_{P}^{2} \vec{B}_{0}^{2}\right)\right) \vec{E}_{0}=-8 \bar{\theta}_{4} \mathcal{L}^{2} \ell_{P}^{2}\left(\left(\vec{k} \times \vec{E}_{0}\right) \cdot \vec{B}_{0}\right)\right)\left(\vec{k} \times \vec{B}_{0}\right) .
$$

Since $\vec{B}_{0}$ and $\vec{k}$ determine a plane, it is natural to study separately the propagation of waves with polarization parallel and perpendicular to this plane. We will express the answer in terms of the refraction index

$$
n=\frac{k c}{\omega} .
$$

¿From (103) we obtain the following refraction indices $(\hbar=1=c)$

$$
\begin{aligned}
& n_{\|}=1-2 \bar{\theta}_{4} \frac{1}{\omega^{2}} \ell_{P}^{2} \vec{B}_{0}^{2}, \\
& n_{\perp}=1-2 \bar{\theta}_{4} \frac{1}{\omega^{2}} \ell_{P}^{2} \vec{B}_{0}^{2}\left(1+2 \sin ^{2} \phi\right),
\end{aligned}
$$

for parallel and perpendicular photon polarization, respectively. Here $\phi$ is the angle between $\vec{k}$ and $\vec{B}_{0}$.

These results can be compared with similar effects in quantum electrodynamics [39]

$$
\begin{gathered}
n_{\|}=1+\theta_{\|} \vec{B}_{0}^{2} \sin ^{2} \phi, \\
n_{\perp}=1+\theta_{\perp} \vec{B}_{0}^{2} \sin ^{2} \phi .
\end{gathered}
$$


where

$$
\theta_{\|}=\frac{2 e^{4}}{45 m^{4}}, \quad \theta_{\perp}=\frac{7 e^{4}}{90 m^{4}} .
$$

As expected, quantum gravity induced effects are much smaller than purely quantum electrodynamics effects, but the former present different signatures. In particular, the indices arising from quantum gravity are frequency dependent and also $n_{\|}$is independent of $\phi$.

\section{Photon time delay}

Notice that our considerations assumed a coarse-grained flat spacetime rather than a FLRW model. The latter would seem more appropriate for GRB traveling cosmological distances. In particular, it would be interesting to study the redshift effects in the photon time delays induced by the energy dependent corrections to the velocity. We are able to estimate these effects as follows.

Using the flat FLRW metric let us calculate the present time delay of two photons emitted simultaneously with different momenta and hence different velocities. We set $r=r_{1}=L, t=t_{1}$ as the emission coordinates and $r=0, t=t_{0}$ as the detection coordinates in the comoving cosmological system $t, r, \theta(t)=0$. The definition of the velocity $V(t)=R(t) \frac{d r}{d t}$, with $\frac{d r}{d t}<0$, leads to

$$
r_{1}=\int_{0}^{r_{1}} d r=\int_{t_{1}}^{t_{0}} \frac{V(t)}{R(t)} d t
$$

Here $R(t)$ is the scale factor in the FLRW metric. Notice that the above equation leads to the standard red shift result for photons moving with $V=c$. On the other hand, quantum gravity corrections predict

$$
\frac{V}{c}=1+\theta\left(\ell_{P} k\right)
$$

to leading order. We are interested in discussing the situation where two photons are emitted at $r_{1}$ with different velocities $V_{1}$ and $V_{1}+\delta V_{1}$, and arrive at $r=0$ at times $t_{0}$ and $t_{0}+\delta t_{0}$ 
respectively. Then we have

$$
r_{1}=\int_{t_{1}}^{t_{0}} \frac{d t}{R(t)} V(t), \quad r_{1}=\int_{t_{1}}^{t_{0}+\delta t_{0}} \frac{d t}{R(t)}(V(t)+\delta V(t)) .
$$

Subtracting the two expressions for the fixed coordinate $r_{1}$, we obtain

$$
\delta t_{0}=-\frac{R_{0}}{V(0)} \int_{t_{1}}^{t_{0}} \frac{d t}{R(t)} \delta V(t)
$$

In the flat FLRW universe we have

$$
t=\frac{t_{0}}{(1+z)^{3 / 2}}, \quad t_{0}=\frac{2}{3} \frac{1}{H_{0}}, \quad d t=-\frac{1}{H_{0}} \frac{1}{(1+z)^{5 / 2}} d z, \quad \frac{R(t)}{R_{0}}=\frac{1}{1+z},
$$

where $z$ is the red shift. This leads to

$$
\delta t_{0}=\frac{1}{c H_{0}} \int_{0}^{z_{1}} \frac{d z}{(1+z)^{3 / 2}} \delta V(z),
$$

where $z_{1}$ is the red shift of the source and $\delta V(z)$ is the difference between the velocities of the two photons, arising from the quantum gravity corrections (109). Using the zeroth-order relation for the red shift of the photon momentum,

$$
k(z)=\frac{R_{0}}{R(z)} k_{0}
$$

we obtain

$$
\delta V(z)=\theta \frac{R_{0}}{R(z)}(\delta k)_{0} \ell_{P}=\theta(1+z)(\delta k)_{0} \ell_{P}
$$

where $(\delta k)_{0}$ is the present day observed momentum difference between the two photons.

Substitution of the above equation in (113) yields

$$
\delta t_{0}=\left(\frac{2 \theta}{H_{0}}\right)(\delta k)_{0} \ell_{P}\left(\left(1+z_{1}\right)^{1 / 2}-1\right) .
$$

The above result differs from the corresponding one obtained in the second and third papers of Ref. [10]. 


\section{Summary and Discussion}

In this paper we have considered the propagation of photons in a semiclassical background provided by loop quantum gravity. An effective electromagnetic Hamiltonian, given by (88), was identified with the expectation value of the electromagnetic piece of the Hamiltonian constraint for the Einstein-Maxwell theory with respect to a would be semiclassical state. The state used was assumed to approximate a classical flat metric, a classical flat gravitational connection and a generic classical electromagnetic field, at scales larger than the coarse-grained characteristic length $\mathcal{L}$, where $\mathcal{L} \gg \ell_{P}$ (the Planck length). To leading non trivial order in $\ell_{P}$, photons of wavelength $\lambda$, where $\mathcal{L}<\lambda$, acquire Planck scale modified dispersion relations as compared to those in classical flat spacetime. This in turn yields the effective speed of light (97) which involves two types of corrections. One of them is just that of Gambini and Pullin [9] including the helicity of the photon, whereas the other depends on the scale $\mathcal{L}$. Moreover, the latter type contains a parameter $\Upsilon$ that encodes the scaling of the gravitational connection under the semiclassical expectation value.

When estimating the coarse-grained characteristic length by $\mathcal{L}=1 / k$, which is its maximal value, the following values of $\Upsilon$ are prominent: (i) $\Upsilon=0$ can be understood as that the connection can not be probed below the coarse graining scale $\mathcal{L}$. The corresponding correction scales as $\left(k \ell_{P}\right)^{2}$. (ii) $\Upsilon=1$ may be interpreted as the analog of the coherent states analysis, where such states saturate the Heisenberg uncertainty relation inside a box of volume $\mathcal{L}^{3}$ : $\Delta q \sim \frac{\ell_{P}}{\mathcal{L}}, \Delta A \sim \frac{\ell_{P}}{\mathcal{L}^{2}}$ and $\Delta q \Delta A \sim \frac{\kappa \hbar}{\mathcal{L}^{3}}$ 38. Then the correction behaves like $\left(k \ell_{P}\right)^{4}$. (iii) Interestingly, a value $\Upsilon=-\frac{1}{2}$ leads to a helicity independent first order correction (i.e. $\left(k \ell_{P}\right)$ ) similar to that of Ellis et al. [10]. We do not have an interpretation of this case though.

A prime candidate for testing the effects which are linear in the energy would be the Gamma Ray Bursts that travel cosmological distances and which might be detected with a time resolution beyond $10^{-5}$ seconds. This seems possible in future spatial experiments [8].

Moreover, new non-linear terms in the Maxwell equations appear. These terms are not 
present either in [9] or [10]. We have explored the significance of this contribution to the propagation of photons in a constant strong magnetic field. The corrections obtained in the corresponding refraction indices are much smaller than similar effects in Quantum Electrodynamics. Nevertheless, quantum gravity corrections have distinct signatures: a main difference is that the speed of photons with polarization parallel to the plane formed by the background magnetic field and the direction of the wave is isotropic.

Our results should be taken as first steps in the exploration of possible observable consequences of quantum gravity. We have given evidence that dispersion relations of the form (1) can have origin in the microstructure of spacetime. It is expected that the recently proposed coherent states for quantum gravity and gauge theories in 36 and/or statistical geometry [37], will come to terms systematically with the unknown numerical coefficients we have left undetermined in our calculation (See also [41].) Interestingly, a quantum field theory in effective spacetimes might possibly emerge along these lines. Yet another avenue in the context of canonical quantization of gravity and gauge theories has recently emerged to understand the semiclassical regime. It is aimed at establishing a relation between Fock space and $\mathcal{H}_{\text {aux }}$ 42, 43.

Further work remains to be done in the framework here developed. For instance, in the case of inflationary cosmology as well as in the study of the Hawking effect, use is made of scalar fields with non standard near Planckian frequency dispersion relations to model the effect of short distance physics on the quantum fields [40. Indeed a systematic study of the modifications induced by quantum gravity along the lines we have developed could be performed to investigate whether the dispersion relations used in [40] can be accounted for.

Finally, we stress that the dispersion relations we have found, as well as those in [9, 10, 20. 21, 40 are Lorentz symmetry violating. This is not necessarily an issue as it has been extensively discussed previously [4, 5]. In fact they may alleviate some long standing astrophysical and cosmological problems [44, 45]. Remarkably, there has been considerable progress in setting bounds to Lorentz invariance violation [46, 47, 48] and to the values of some coefficients in the 
effective Maxwell equations [49].

Acknowledgments. The authors are grateful to A. Ashtekar, M. Bojowald, R. Gambini and T. Thiemann for illuminating comments at different stages of this work. Partial support is acknowledged from CONICyT-CONACyT E120-2639, DGAPA IN11700 and CONACYT 32431-E. We also acknowledge the project Fondecyt 7010967. The work of JA is partially supported by Fondecyt 1010967. HAMT thanks for the warm hospitality and stimulating environment experienced at the Center for Gravitational Physics and Geometry-PSU, in particular to A. Ashtekar and J. Pullin. He also acknowledges partial support from NSF Grants PHY0090091 and INT97-22514, Eberly Research Funds at Penn State, CONACyT 55751 and MéxicoUSA Foundation for Science. LFU acknowledges the hospitality at the Center for Gravitational Physics and Geometry-PSU.

\section{References}

[1] G. Amelino-Camelia, J. Ellis, N.E. Mavromatos, D.V. Nanopoulos and S. Sarkar, Tests of quantum gravity from observations of $\gamma$-ray bursts, Nature 393 (1998) 763-765.

[2] J. Ellis, N.E. Mavromatos and D.V. Nanopoulos, Search for quantum gravity, Gen. Rel. Grav. 31 (1999) 1257 gr-qc/9905048.

[3] D.V. Ahluwalia, Quantum gravity: testing times for theories, Nature 398 (1999) 199; G.Z. Adunas, E. Rodriguez-Milla and D.V. Ahluwalia, Probing quantum aspects of gravity, Phys. Letts. B485(2000)215-223, gr-qc/0006021; G.Z. Adunas, E. Rodriguez-Milla and D.V. Ahluwalia, Probing quantum violations of the equivalence principle, Gen. Rel. Grav. 33(2001)183-194, [gr-qc/0006022]; D.V. Ahluwalia, C.A. Ortiz and G.Z. Adunas, Robust flavor equalization of cosmic neutrino flux by quasi bimaximal mixing, hep-ph/0006092. 
[4] G. Amelino-Camelia, Are we at the dawn of quantum gravity phenomenology?, Lectures given at 35th Winter School of Theoretical Physics: From Cosmology to Quantum Gravity, Polanica, Poland, 2-12 Feb 1999. Published in Lect. Notes Phys. 541 (2000) 1-49, Springer Verlag [gr-qc/9910089].

[5] G. Amelino-Camelia, Planck length phenomenology, Int. Jour. Mod. Phys. D10 (2001) 1, gr-qc/0008010.

[6] J. van Paradis et. al., Transient optical emission from the error box of the $\gamma$-ray burst of 28 february 1997, Nature 386(1997)686; P.J. Groot et al.,IAU Circ. No 6676, 1997; M. L. Metzger et al., Spectral constraints on the red shift of the optical counterpart of the $\gamma$-ray burst of 8 May 1997, Nature 387 (1997)878.

[7] P.N. Bhat, G.J. Fishman, C.A. Meegan, R.B. Wilson, M.N. Brock and W.S. Paclesas, Evidence for sub-millisecond structure in a $\gamma$-ray burst, Nature 359(1992)217.

[8] P. Mészáros, Gamma-Ray bursts and bursters, Nucl. Phys. B (Proc. Suppl.) 80 (2000) 63-77.

[9] R. Gambini and J. Pullin, Non standard optics from quantum spacetime, Phys. Rev. D59 (1999) 124021, gr-qc/9809038.

[10] J. Ellis, N.E. Mavromatos and D.V. Nanopoulos, Quantum gravitational difussion and stochastic fluctuations in the velocity of light, Gen. Rel. Grav. 32 (2000) 127-144, grqc/9904068]; J. Ellis, N.E. Mavromatos and D.V. Nanopoulos, Tegernsee 1999, Beyond the desert, p.299-334, gr-qc/9909085 and references therein; J. Ellis, K. Farakos, N.E. Mavromatos, V.A. Mitsou and D.V. Nanopoulos, Astrophysical Probes of the Constancy of the Velocity of Light, Astrophysical Jour. 535 (2000) 139-151, astro-ph/9907340.

[11] N.E. Mavromatos, The quest for quantum gravity: testing times for theories?, astroph/0004225]; J. Ellis, Perspectives in High-Energy Physics, JHEP Proceedings, SILAFAE 
III, Cartagena de Indias, Colombia, april 2-8,2000, hep-ph/0007161]; J. Ellis, Testing fundamental physics with high-energy cosmic rays, astro-ph/0010474.

[12] D.A.R. Dalvit, F.D. Mazzitelli, C. Molina-Paris, One loop graviton corrections to Maxwell's equations, Phys. Rev. D63(2001) 084023, hep-th/0010229.

[13] F. Benatti and R. Floreanini, Massless neutrino oscillations, hep-ph/0105303; Effective dissipative dynamics for polarized photons, Phys. Rev. D62 (2000) 125009, hep$\mathrm{ph} / 0009283$.

[14] H. Yu and L.H. Ford, Lightcone fluctuations in flat spacetimes with non trivial topology, Phys. Rev. D60 (1999) 084023, gr-qc/9904082].

[15] E. Waxman and J. Bahcall, High energy neutrinos from cosmological gamma ray burst fireballs, Phys. Rev. Letts. 78(1997) 2292-2295; E. Waxman, High energy neutrinos from gamma ray bursts, Nucl. Phys. (Proc. Supl) 91 (2000) 494-500 ; Gamma ray bursts, cosmic rays and neutrinos, Nucl. Phys. (Proc. Supl) 87 (2000) 345-354.

[16] M. Vietri, Ultrahigh-energy neutrinos from gamma ray bursts, Phys. Rev. Letts. 80(1998) 3690-3693.

[17] M. Roy, H.J. Crawford and A. Trattner, The prediction and detection of UHE Neutrino Bursts, astro-ph/9903231.

[18] D.B. Cline and F.W. Stecker, Exploring the ultrahigh energy neutrino universe, astro$\mathrm{ph} / 0003459$.

[19] F. Halzen, High-energy neutrino astronomy, Phys. Rep. 333 (2000) 349-364.

[20] J. Alfaro, H.A. Morales-Técotl and L.F. Urrutia, Quantum gravity corrections to neutrino propagation, Phys. Rev. Letts. 84(2000)2318, [gr-qc/9909079]. 
[21] J. Ellis, N.E. Mavromatos, D.V. Nanopoulos and G. Volkov, Gravitational recoil effects of fermion propagation in spacetime foam, Gen. Rel. Grav. 32 (2000) 1777-1798, grqc/9911055].

[22] C. Rovelli and L. Smolin, Discreteness of area and volume in quantum gravity, Nucl. Phys. B442 (1995) 593-622; Erratum-ibid B456 (1995) 753. A. Ashtekar and J. Lewandowsky, Quantum theory of geometry I: Area operators, Class. Quant. Grav. 14 (1997) A55A82; Quantum theory of geometry II: Volume operators, Adv. Theor. Math. Phys. 1 (1998) 388-429. A. Ashtekar, A. Corichi and J. Zapata, Quantum theory of geometry III: Noncommutativity of Riemannian structures, Class. quant. Grav. 15 (1998) 2955-2972.

[23] C. Rovelli, Loop quantum gravity and black hole physics, Helv. Phys. Acta 69 (1996) 582611 gr-qc/9608032. C. Rovelli, Black hole entropy from loop quantum gravity, Phys. Rev. Lett.77 (1996) 3288-3291,1996 gr-qc/9603063. A. Ashtekar, J. Baez, A. Corichi and K. Krasnov, Quantum geometry and black hole entropy, Phys. Rev. Lett. 80 (1998) 904-907 gr-qc/9710007.

[24] M. Bojowald, Absence of singularity in loop quantum cosmology, Phys. Rev. Lett. 86 (2001) 5227-5230 gr-qc/0102069.

[25] For a recent review see for example C. Rovelli, Loop quantum gravity, Livings Reviews Vol 1, 1998-1, http://www.livingreviews.org /Articles. See also R. Gambini and J. Pullin, Loops, Knots, Gauge Theories and Quantum Gravity, Cambridge University Press, Cambridge UK 1996.

[26] A. Ashtekar, New variables for classical and quantum gravity, Phys. Rev. Letts. 57 (1987) 2244. C. Beetle and A. Corichi, Bibliography of publications related to classical and quantum gravity in terms of connections and loop variables gr-qc/9703044. 
[27] A. Ashtekar, J. Lewandowski, D. Marolf, J. Mourao and T. Thiemann, Quantization of diffeomorphism invariant theories of connections with local degrees of freedom, Jour. Math. Phys. 36 (1995) 6456-6493 gr-qc/9504018.

[28] F. Barbero, Real Ashtekar variables for Lorentzian signature space times, Phys. Rev. D51 (1995) 5507-5510; Reality conditions and Ashtekar variables: a different perspective, Phys. Rev. D51 (1995) 5498-5506.

[29] T. Thiemann, Reality conditions inducing transforms for quantum gauge field theory and quantum gravity, Class. Quant. Grav. 13 (1996) 1383-1404 gr-qc/9511057. Ashtekar, A generalized Wick transform for gravity, Phys. Rev. D53 (1996) 2865-2869 [gr-qc/9511083]. A. Ashtekar, J. Lewandowski, D. Marolf, J. Mourao, T. Thiemann, Coherent state transforms for spaces of connections, J. Funct. Anal. 135 (1996) 519-551 gr-qc/9412014.

[30] H.A. Morales-Técotl, L.F. Urrutia, J.D. Vergara, Reality conditions for Ashtekar variables as Dirac constraints, Class. Quant. Grav. 13 (1996) 2933-2940 [gr-qc/9607044]. M. Montesinos, H.A. Morales-Tecotl, L.F. Urrutia and J.D. Vergara, Real sector of the nonminimally coupled scalar field to selfdual gravity, J. Math. Phys. 40 (1999) 1504-1517 gr-qc/9903043; Complex canonical gravity and reality constraints, Gen. Rel. Grav. 31 (1999) 719-723.

[31] T. Thiemann, QSD5: Quantum gravity as the natural regulator of matter quantum field theories, Class. Quan. Grav. 15 (1998) 1281-1314; Quantum Spin Dynamics (QSD), Class. Quant. Grav. 15 (1998) 839-873.

[32] A. Ashtekar, C. Rovelli and L. Smolin, Weaving quantum geometries with quantum threads, Phys. Rev. Letts. 69(1992) 237-240, hep-th/9203079]. J. Zegwaard, The weaving of curved geometries, Phys. Lett. B300 (1993) 217-222 hep-th/9210033. R. Borissov, Weave states for plane gravitational waves, Phys. Rev. D49 (1994) 923-929. J. Iwasaki and 
C. Rovelli, Gravitons as embroidery on the weave, Int. J. Mod. Phys. D 1 (1993) 533-557; Gravitons from loops: nonperturbative loop space quantum gravity contains the graviton physics information, Class. and Quant. Grav. 11 (1994) 1653-1676. J. Iwasaki, Basis states for gravitons in nonperturbative loop representation space gr-qc/9807013].

[33] B. Brugmann, R. Gambini and J. Pullin, Knot invariants as nondegenerate quantum geometries, Phys. Rev. Letts. 68 (1992) 431-434.

[34] N. Grot and C. Rovelli, Weave states in loop quantum gravity, Gen. Rel. Grav. 29 (1997) 1039-1048.

[35] A. Corichi and J.M. Reyes, A Gaussian weave for kinematical loop quantum gravity, Int. J. Mod. Phys. D 10 (2001) 325-338 [gr-qc/0006067.

[36] T. Thiemann, Gauge field theory coherent states (GCS) I: General properties, Class. Quant. Grav. 18 (2001) 2025-2064, hep-th/0005233; T. Thiemann and O. Winkler, Gauge field theory coherent states (GCS) II: Peakedness properties, Class. Quant. Grav. 18 (2001) 2561-2636, hep-th/0005237. T. Thiemann and O. Winkler, Gauge field theory coherent states (GCS): III. Ehrenfest theorems [hep-th/0005234]; Gauge field theory coherent states (GCS): IV. Infinite tensor product and thermodynamical limit hep-th/0005235. H. Sahlmann, T. Thiemann, O. Winkler, Coherent States for Canonical Quantum General Relativity and the Infinite Tensor Product Extension gr-qc/0102038.

[37] A. Ashtekar and L. Bombelli, Statistical geometry of quantum spin networks: flat space, in preparation; L. Bombelli, Statistical geometry of random weave states, gr-qc/0101080.

[38] For the analogous situation in electrodynamics see for example W. Heitler, Quantum Theory of Radiation, $3^{\text {rd }}$ edition, Clarendon Press, Oxford, England (1954).

[39] L.D. Landau and E.M. Lifshitz, Quantum Electrodynamics, Pergamon Press, 1967, page 585. 
[40] T. Jacobson and D. Mattingly, Generally covariant model of a scalar field with high frequency dispersion and the cosmological horizon problem, Phys. Rev. D63 (2001) 041502(R); T. Jacobson, Lorentz violation and Hawking radiation, gr-qc/0110079.

[41] M. Varadarajan and J.A. Zapata, A proposal for analyzing the classical limit of kinematic loop quantum gravity, Class. Quant. Grav. 17 (2000) 4085-4110 gr-qc/0001040.

[42] M. Varadarajan, Fock representations from U(1) holonomy algebras, Phys. Rev. D 61 (2000) 104001 gr-qc/0001050; Photons from quantized electric flux representations grqc/0104051].

[43] A. Ashtekar and J. Lewandowski, Relation between polymer and Fock excitations, Class.Quant.Grav. 18L117-L128(2001), gr-qc/0107043.

[44] G. Amelino-Camelia, Space-time quantum solves three experimental paradoxes, grqc/0107086].

[45] J. Alfaro and G. Palma, Loop Quantum Gravity corrections and Cosmic Rays decays, hep-th/0111176.

[46] D. Colladay and V.A. Kostelecky, Lorentz-violating extension of the Standard Model, Phys. Rev. D58 (1998) 116002 hep-ph/9809521]. For a recent review see for example V.A. Kostelecky, Topics in Lorentz and CPT violation, hep-ph/0104227 and references therein.

[47] J.M. Carmona and J.L. Cortés, Testing Lorentz invariance in the Tritium Beta decay anomaly, Phys. Lett. B 494(2000)75-80 hep-ph/0007057; Infrared and ultraviolet cutoffs of quantum field theory [hep-th/0012028]. 
[48] S. Liberati, T. Jacobson and D. Mattingly, High-energy constraints on Lorentz symmetry violations, hep-ph/0110094; T. Jacobson, Stefano Liberati and D. Mattingly, TeV astrophysics constraints on Planck scale Lorentz violation, hep-ph/0112207.

[49] R.J. Gleiser and C.N. Kozameh, Astrophysical limits on quantum gravity motivated birefringence, Phys. Rev. D64083007 (2001), gr-qc/0102093. 